\title{
ON THE BULK VELOCITY OF BROWNIAN RATCHETS
}

\author{
STANISLAV KONDRATYEV, JOSÉ MIGUEL URBANO, \\ AND DMITRY VOROTNIKOV
}

\begin{abstract}
In this paper we study the unidirectional transport effect for Brownian ratchets modeled by Fokker-Planck-type equations. In particular, we consider the adiabatic and semiadiabatic limits for tilting ratchets, generic ratchets with small diffusion, and the multi-state chemical ratchets. Having established a linear relation between the bulk transport velocity and the bi-periodic solution, and using relative entropy estimates and new functional inequalities, we obtain explicit asymptotic formulas for the transport velocity and qualitative results concerning the direction of transport. In particular, we prove the conjecture by Blanchet, Dolbeault and Kowalczyk that the bulk velocity of the stochastic Stokes' drift is non-zero for every non-constant potential.
\end{abstract}

\section{INTRODUCTION}

Brownian ratchets or Brownian motors are generic terms for tiny devices which are able to produce unidirectional transport of matter when all acting forces and gradients vanish after averaging over space and time, and at the presence of (and often due to) overdamped Brownian motion [2, 15, 19, 27, 32, 33]. Motor proteins are generally considered to be the most celebrated example of Brownian ratchets [1]. However, during recent years there has been a huge progress [18] in realizing and observing bulk motion without net bias in SQUIDs, Josephson junctions, cold atoms in optical lattices, nanopores, etc., as well as in microfluidics experiments. The Stokes' drift with diffusion is also an example of a Brownian ratchet mechanism [4, 19, 21]. Although the idea

2010 Mathematics Subject Classification. 26D10; 35Q84; 35Q92; 47H10; 60J70.

Key words and phrases. Brownian motor, tilting ratchet, stochastic Stokes' drift, Fokker-Planck equation, periodic solution, transport, relative entropy.

The third author is grateful to Danielle Hilhorst, Steven Lade, Rafael Ortega and Fabio Zanolin for very useful correspondence. The research was partially supported by CMUC, funded by COMPETE and FCT under the project PEst-C/MAT/UI0324/2013, and by FCT projects UTA-CMU/MAT/0007/2009 and PTDC/MAT-CAL/0749/2012. 
of micro-level motors goes back to the dawn of thermodynamics, the discovery of ratchets has boosted the contemporary nano-technological interest in the development of hybrid and artificial molecular machines [7, [22].

The dynamics of a ratchet can be described by the Fokker-Planck equation

$$
\rho_{t}-\sigma \rho_{x x}-\left(\Psi_{x} \rho\right)_{x}=0,
$$

where $\sigma$ is the given diffusion coefficient, and $\rho(x, t)$ is the unknown probability density of distribution of Brownian particles governed by a given potential $\Psi(x, t)$, which is supposed to be $T$-periodic in time $t$, and to have a 1-periodic in $x$ derivative $\Psi_{x}(x, t)$. Note that we do not assume the potential $\Psi(x, t)$ itself to be $x$-periodic, so various tilting regimes are allowed, and the 'tilting forces' are contained within the potential.

A related model which has particular relevance in biological applications is the chemical motor. Here the particles can be in several states, and the total amount of particles is fixed. Particles in different states are sensitive to different time-independent potentials. The underlying chemical processes cause transitions between the particles' states, which we can consider to be random. This is described by the following system of Fokker-Planck-type equations:

$$
\left(\rho_{i}\right)_{t}-\sigma\left(\rho_{i}\right)_{x x}-\left(\left(\Psi_{i}\right)_{x} \rho_{i}\right)_{x}+\sum_{j, j \neq i} \nu_{j i} \rho_{i}=\sum_{j, j \neq i} \nu_{i j} \rho_{j}, i=1, \ldots, N
$$

where $\Psi_{i}(x)$ are the given potentials, and $\sigma$ is the diffusion coefficient (for definiteness, we set it to be the same for all states). We assume that $\left(\Psi_{i}\right)_{x}(x)$ (not the $\Psi_{i}$ themselves) and the transition rates $\nu_{i j}(x)$ are 1-periodic.

Various particular cases of (1.1) and (1.2), including the so-called flashing ratchets, were studied in [5, 6, 8, 9, 13, 20, 23, 24, 28, 29, 30, 36, 37]. To catch the motor effect, the majority of these papers consider (1.1) or (1.2) with no-flux boundary conditions on a bounded segment, and show, under appropriate assumptions, that the mass is eventually concentrated closer to one edge of the segment than to the other. Yet equation (1.1) with the travelling potential $\Psi(x, t)=\psi(x-\omega t)$ and the flashing potential $\Psi(x, t)=h(t) \psi(x)$, where $\psi$ is 1-periodic, and

$$
h(t)= \begin{cases}1 & \text { if } k T<t \leq(k+1 / 2) T, \\ 0 & \text { if }(k+1 / 2) T<t \leq(k+1) T, \quad(k=0,1, \ldots)\end{cases}
$$

was examined on the whole real line in [5, 6] and [24, respectively. In [24] it was observed that the solutions of a homogenized equation tend 
to propagate with constant speed or not to move at all as the period length goes to zero. In [5, 6] it was shown that with the course of time the velocity of the centre of mass eventually becomes the same for all solutions (see also [12]). Moreover, this asymptotic speed is equal to

$$
v_{\infty}=-\frac{1}{T} \int_{0}^{T} \int_{0}^{1} \Psi_{x}(x, t) g_{\infty}(x, t) d x d t,
$$

where $g_{\infty}$ is the bi-periodic (in $x$ and $t$ ) travelling wave solution to (1.1).

In this paper, we develop a unified approach for detecting transport for generic equations (1.1) and (1.2). We prove that the averaged velocity stabilizes as time goes to infinity, and the limiting velocity is independent of the solution. We establish a linear relation between this velocity and a certain solution to (1.1) or (1.2), respectively. This solution is actually the bi-periodic solution in the case of (1.1), and is the stationary $x$-periodic solution vector in the case of (1.2). This allows us to obtain a more explicit characterization of the occurrence of unidirectional transport, its direction and bulk velocity, for 1- and 2 -state tilting ratchets, in the adiabatic and semiadiabatic regimes, for the stochastic Stokes' drift, and for generic low-diffusion-driven 1- and 2-state ratchets (1.1) and (1.2). We also prove the conjecture stated by Blanchet, Dolbeault, and Kowalczyk in [5] (see also [32]) that the bulk velocity of the stochastic Stokes' drift is non-zero for every nonconstant potential.

The paper is organized as follows. In Section 2 we set the framework for our research. In particular, we define the transport in terms of the asymptotic average bulk velocity and relate it to the bi-periodic solution of an auxiliary space-periodic problem.

In Section 3 we consider the adiabatic regime for tilting ratchets, i.e., we suppose that the ratchet spends a long time in each of its states. In Theorem 3.1 we justify the explicit formula (3.8) for the adiabatic transport velocity. Developing this topic, we state an effective formula for the direction of transport (Proposition 3.8). Finally, Theorem 3.12 gives a qualitative result showing that a major interval of monotonicity of the potential implies a particular direction of transport. A highlight of Section 3 is Proposition 3.14 establishing a nontrivial functional inequality.

In Section 4 we study the so-called semiadiabatic regime for tilting ratchets, when the overall period of tilting goes to infinity and one of the tilting states dominates the other. We give an effective explicit formula (4.5) for the semiadiabatic transport velocity and prove that non-constant potentials produce nonzero semiadiabatic transport in 
one and the same direction-Theorem 4.4 and Corollaries 4.5 and 4.6 . These results are partially based on the functional inequality related with Proposition 4.1, which also implies the conjecture of [5] (see also [32]) that the stochastic Stokes' drift generates unidirectional transport for every non-constant potential.

In Section 5 we consider generic Brownian ratchets with small diffusion coefficient and show that there is a connection between the transport and a certain ODE. We show (Theorem 5.1) that if this ODE does not have periodic solutions or, equivalently, if its Poincaré rotation number in nonzero, there appears directed transport of mass.

In Section 6 we extend our approach to multi-state models. The most interesting results are obtained for the case of small diffusion: Theorem 6.3 classifies it with respect to the geometry of zeroes of the potential gradients and establishes the direction of transport in different cases. Finally, Theorem 6.6 treats the adiabatic and semiadiabatic regimes for the randomly tilting ratchet.

\section{BulK VELOCITy}

2.1. Unidirectional transport. We model the dynamics of a ratchet by the Fokker-Planck equation

$$
\left\{\begin{array}{l}
\rho_{t}-\sigma \rho_{x x}-\left(\Psi_{x} \rho\right)_{x}=0, \quad x \in \mathbb{R}, t>0, \\
\rho=\rho_{0}(x), \quad x \in \mathbb{R} .
\end{array}\right.
$$

Here $\Psi(x, t)$ is a given potential, and $\rho_{0}$ is a given initial condition. We assume that $\Psi(x, t)$ is $T$-periodic in $t$ and its derivative $\Psi_{x}(x, t)$ is 1-periodic in $x$, and we also assume that $\rho_{0}(x)$ satisfies the requirements

$$
\rho_{0}(x) \geq 0, \quad \int_{-\infty}^{\infty} \rho_{0}(x) d x=1, \quad \int_{-\infty}^{\infty}|x| \rho_{0}(x) d x<\infty .
$$

We are interested in nonnegative solutions of (2.1). Such solutions may be viewed as non-stationary distributions of a unit mass on $\mathbb{R}$ whose movement is governed by a potential force and by diffusion.

The most interesting cases arise when $\Psi$ is 'unbiased' in the sense that

$$
\int_{0}^{T} \int_{0}^{1} \Psi_{x}(x, t) d x d t=0,
$$

i.e., the time and space average of the potential gradient vanishes. However, for the sake of generality, in the sequel we do not assume (2.3) unless explicitly specified.

It can be proved by classical methods (see e.g. 31]) that if $\Psi$ is continuous in $(x, t)$ and $C^{2, \alpha}$-regular in $x$, where $\alpha \in(0,1)$ is independent 
of $t$, then (2.1) is uniquely solvable for any continuous initial data $\rho_{0}$ satisfying (2.2); moreover, the solution $\rho(x, t)$ is positive for any $t>0$, and

$$
\lim _{x \rightarrow \pm \infty}|x|\left(|\rho(x, t)|+\left|\rho_{x}(x, t)\right|\right)=0 .
$$

A consequence of (2.4) is the conservation of mass

$$
\int_{-\infty}^{\infty} \rho d x=1
$$

and the finiteness of the centre of mass

$$
\bar{x}(t)=\int_{-\infty}^{\infty} x \rho(x, t) d x
$$

for any $t$. We study the asymptotic behaviour of solutions of (2.1) as $t \rightarrow \infty$. Properties (2.4), (2.5), and (2.6) are crucial for our approach.

To catch the unidirectional transport effect, we consider the velocity of the centre of mass $\bar{x}(t)$, which is called the drift (or bulk, or ballistic) velocity. Specifically, we consider the average drift velocity on the interval $[t, t+T]$, and if it has a nonzero limit as $t \rightarrow+\infty$, we say we have unidirectional transport.

Due to the periodic nature of the problem at issue, the drift velocity is conveniently characterized by means of the following problem on the circle $S^{1}=\mathbb{R} / \mathbb{Z}$ :

$$
\begin{aligned}
& g_{t}-\sigma g_{x x}+(F g)_{x}=0, \quad(x, t) \in S^{1} \times(0, \infty) \\
& g(x, 0)=g_{0}(x), \quad x \in S^{1} ; \quad g_{0}(x) \geq 0, \quad \int_{S^{1}} g_{0}(x) d x=1 .
\end{aligned}
$$

Here $F(x, t)$ is defined on $S^{1} \times(0, \infty)$; generally we assume that it is $T$-periodic in $t$.

To start with, observe that if $\rho$ is a nonnegative solution of (2.1), then, by linearity, the nonnegative function

$$
g(x, t)=\sum_{k=-\infty}^{\infty} \rho(x+k, t)
$$

solves (2.7) with $F=-\Psi_{x}$. 
Consider the average velocity of the centre of mass as $t$ varies from $t_{0}$ to $t_{0}+T$ :

$$
\begin{aligned}
& v_{\left[t_{0}, t_{0}+T\right]}:= \frac{\bar{x}\left(t_{0}+T\right)-\bar{x}\left(t_{0}\right)}{T} \\
&= \frac{1}{T}\left(\int_{-\infty}^{\infty} x \rho\left(x, t_{0}+T\right) d x-\int_{-\infty}^{\infty} x \rho\left(x, t_{0}\right) d x\right) \\
&=\frac{1}{T} \int_{-\infty}^{\infty} x \int_{t_{0}}^{t_{0}+T} \rho_{t}(x, t) d t d x \\
&=\frac{1}{T} \int_{t_{0}}^{t_{0}+T} \int_{-\infty}^{\infty} x\left(\sigma \rho_{x x}+\left(\Psi_{x} \rho\right)_{x}\right) d x d t \\
&= \frac{1}{T} \int_{t_{0}}^{t_{0}+T}\left(-\sigma \int_{-\infty}^{\infty} \rho_{x} d x-\int_{-\infty}^{\infty} \Psi_{x} \rho d x\right) d t \\
&=-\frac{1}{T} \int_{t_{0}}^{t_{0}+T} \sum_{k=-\infty}^{\infty} \int_{0}^{1} \Psi_{x}(x+k, t) \rho(x+k, t) d x d t \\
&=-\frac{1}{T} \int_{t_{0}}^{t_{0}+T} \int_{0}^{1} \Psi_{x}(x, t) g(x, t) d x d t
\end{aligned}
$$

(here we have used (2.4) and the periodicity of $\Psi_{x}$ ). Thus we have the following formula for the average drift velocity:

$$
v_{\left[t_{0}, t_{0}+T\right]}=-\frac{1}{T} \int_{t_{0}}^{t_{0}+T} \int_{0}^{1} \Psi_{x}(x, t) g(x, t) d x d t .
$$

Remark 2.1. We point out that the conservation of mass holds for (2.7)-(2.8), i.e.,

$$
\int_{S^{1}} g(x, t) d x=1
$$

for any solution thereof.

2.2. Bi-periodic solution. The notion of relative entropy and related inequalities are a useful tool for the study of the Fokker-Planck equation (2.7).

Given $g, h \in L_{+}^{1}\left(S^{1}\right)$ such that

$$
\int_{S^{1}} g d x=1=\int_{S^{1}} h d x
$$

define the relative entropy of $g$ with respect to $h$ by

$$
\mathrm{e}[g \mid h]=\int_{S^{1}} g \ln \frac{g}{h} d x
$$


Observe that $\mathrm{e}[g \mid h] \geq 0$ (the possibility $\mathrm{e}[g \mid h]=\infty$ is not excluded). Indeed, letting $r=g / h \geq 0$ we have

$$
\begin{aligned}
\mathrm{e}[g \mid h]=\int_{S^{1}} g \ln r d x=\int_{S^{1}}(g \ln r-g & +h) d x \\
& =\int_{S^{1}} h(r \ln r-r+1) d x \geq 0
\end{aligned}
$$

as $r \ln r-r+1=\int_{1}^{r} \ln \xi d \xi \geq 0$. Moreover, $\mathrm{e}[g \mid h]=0$ if and only if $g=h$ almost everywhere. This follows from the definition and from the fact that the relative entropy controls $L^{1}$ distance. Specifically, for probability densities $g, h \in L_{+}^{1}\left(S^{1}\right)$ we have the known CsiszárKullback inequality [11]:

$$
\|g-h\|_{L^{1}\left(S^{1}\right)}^{2} \leq 2 \mathrm{e}[f \mid g]
$$

which holds for any probability densities $g, h \in L_{+}^{1}\left(S^{1}\right)$.

Another important tool is the Log-Sobolev inequality [17], which we need in the following form: given $h \in L^{1}\left(S^{1}\right)$ such that

$$
\int_{S^{1}} h d x=1, \quad 0<C_{1} \leq h(x) \leq C_{2} \text { for a. a. } x \in S^{1},
$$

there exists $b=b\left(C_{1}, C_{2}\right)>0$ such that

$$
\int_{S^{1}} g \ln \frac{g}{h} d x \leq b \int_{S^{1}} g\left|\left(\ln \frac{g}{h}\right)_{x}\right|^{2} d x
$$

for any probability density $g \in L_{+}^{1}\left(S^{1}\right)$ such that the derivative on the right-hand side exists almost everywhere. Observe that both sides in (2.13) are nonnegative (possibly infinite) whenever the derivative of the logarithm makes sense.

The integral

$$
\mathrm{I}[g \mid h]=\int_{S^{1}} g\left|\left(\ln \frac{g}{h}\right)_{x}\right|^{2} d x
$$

on the right-hand side of (2.13) is called the entropy production term of $g$ and $h$. Thus (2.13) can be equivalently expressed as

$$
\mathrm{e}[g \mid h] \leq b \mathrm{I}[g \mid h]
$$

The following theorem sets the framework for our investigation of transport. It establishes the existence of a unique time-periodic solution of (2.7), which attracts other solutions and thus is our natural object of study (cf. [13, 6, 3]).

Theorem 2.2. Suppose that $F: S^{1} \times \mathbb{R}_{+}$is T-periodic in the second argument and there exists a partition $0=t_{0}<t_{1}<\cdots<t_{n}=T$ of the segment $[0, T]$ such that $F$ is $C^{3}$ on every segment $\left[t_{i-1}, t_{i}\right]$. Then there 
exists a unique positive $T$-periodic in $t$ solution $g_{\infty}$ of (2.7) satisfying (2.11). Moreover, if $g$ solves (2.7)-(2.8) with the initial condition satisfying $\int_{S_{1}} g_{0} \ln g_{0} d x<\infty$, then $g_{\infty}$ attracts $g$ in the sense of the entropy

$$
\mathrm{e}\left[g(\cdot, t) \mid g_{\infty}(\cdot, t)\right] \leq \mathrm{e}\left[g(\cdot, 0) \mid g_{\infty}(\cdot, 0)\right] \mathrm{e}^{-\gamma t}, t \geq 0,
$$

where $\gamma=\sigma / b$ and $b=b\left(\min g_{\infty}, \max g_{\infty}\right)$ is the uniform Log-Sobolev constant for $g_{\infty}$.

Remark 2.3. If $F$ has discontinuities at the points $t_{i}$, we do not require that (2.7) should hold at these points. In this case we construct solutions piecewise: given the initial data at $t=t_{0}$, by parabolic regularity the solution is well defined at $t=t_{1}$, then $g\left(\cdot, t_{1}\right)$ is considered as the initial condition for the segment $\left[t_{1}, t_{2}\right]$, and so on.

Proof of Theorem 2.2. First suppose that $F$ is $C^{3}$ on $S^{1} \times[0, T]$. Let

$$
X=\left\{g \in L_{+}^{1}\left(S^{1}\right) \mid \int_{S^{1}} g(x) d x=1\right\} .
$$

Consider the operator $\mathcal{T}: X \rightarrow X$ that takes $g_{0} \in X$ to $g(\cdot, T)$, where $g$ solves (2.7)-(2.8). The operator is well defined due to the conservation of mass and the maximum principle. As $X$ is convex and bounded in $L^{1}\left(S^{1}\right)$, its image $\mathcal{T}(X)$ is precompact in $X$ by parabolic regularity. Hence, by the Schauder theorem, $\mathcal{T}$ has a fixed point, which clearly is the initial condition for a $T$-periodic in time solution $g_{\infty}$ of (2.7). We have yet to prove that it is the only periodic solution. By parabolic regularity, $g_{\infty}$ is continuous on $S^{1} \times[0, T]$ and hence bounded, and by the strong maximum principle it is positive and thus bounded away from 0 .

Now suppose $g$ solves (2.7) with the initial condition $g_{0}(x) \geq 0$ such that $\int_{S^{1}} g_{0}(x) \ln g_{0}(x) d x<\infty$. We claim that the relative entropy $\mathrm{e}\left[g(\cdot, t) \mid g_{\infty}(\cdot, t)\right]$ decreases. Indeed, letting $r=g / g_{\infty}$ we have

$$
\begin{aligned}
& \frac{d \mathrm{e}\left[g \mid g_{\infty}\right]}{d t}=\frac{d}{d t} \int_{S^{1}} g \ln \frac{g}{g_{\infty}} d x \\
& =\int_{S^{1}} g_{t} \ln \frac{g}{g_{\infty}} d x+\int_{S^{1}} g_{t} d x-\int_{S^{1}} \frac{g\left(g_{\infty}\right)_{t}}{g_{\infty}} d x \\
& \quad=\int_{S^{1}}\left(\sigma g_{x x}-(F g)_{x}\right) \ln r d x-\int_{S^{1}}\left(g_{\infty}\right)_{t} r d x \\
& =\int_{S^{1}} \frac{g}{r}\left(\sigma r_{x x}+F r_{x}\right) d x-\sigma \int_{S^{1}} g \frac{r_{x}^{2}}{r^{2}} d x-\int_{S^{1}}\left(g_{\infty}\right)_{t} r d x \\
& =\int_{S^{1}} g_{\infty}\left(\sigma r_{x x}+F r_{x}\right) d x-\sigma \int_{S^{1}} g \frac{r_{x}^{2}}{r^{2}} d x-\int_{S^{1}}\left(g_{\infty}\right)_{t} r d x
\end{aligned}
$$




$$
\begin{gathered}
=\int_{S^{1}}\left(\sigma\left(g_{\infty}\right)_{x x}-\left(F g_{\infty}\right)_{x}-\left(g_{\infty}\right)_{t}\right) r d x-\sigma \int_{S^{1}} g \frac{r_{x}^{2}}{r^{2}} d x \\
=-\sigma \int_{S^{1}}\left(\ln \frac{g}{g_{\infty}}\right)_{x}^{2} g d x=-\sigma \mathrm{I}\left[g \mid g_{\infty}\right] .
\end{gathered}
$$

As $g_{\infty}$ is bounded away from 0 , as well as from above for $(x, t) \in$ $S^{1} \times[0, T]$, we have the uniform in $t$ Log-Sobolev inequality

$$
\mathrm{e}\left[g \mid g_{\infty}\right] \leq b \mathrm{I}\left[g \mid g_{\infty}\right]
$$

Hence

$$
\frac{d \mathrm{e}\left[g \mid g_{\infty}\right]}{d t} \leq-\frac{\sigma}{b} \mathrm{e}\left[g \mid g_{\infty}\right]
$$

and (2.15) follows. Moreover, the attraction (2.15) implies the uniqueness of the periodic solution.

In the general case of piecewise continuous $F$, the proof works with slight modifications. We have $\mathcal{T}=\mathcal{T}_{n} \circ \cdots \circ \mathcal{T}_{1}$, where $\mathcal{T}_{i}$ is the resolving operator for the segment $\left[t_{i-1}, t_{i}\right]$, which is compact, so we still can apply the Schauder theorem and obtain a fixed point and a periodic solution. The relative entropy e $\left[g \mid g_{\infty}\right]$ is continuous and the computation (2.16) holds in each open interval $\left(t_{i-1}, t_{i}\right)$, so e $\left[g \mid g_{\infty}\right]$ decreases. Inequality (2.17) also holds in each open interval $\left(t_{i-1}, t_{i}\right)$, whence (2.15) follows. Indeed, for $t_{1}$ we have

$$
\left.\mathrm{e}\left[g \mid g_{\infty}\right]\right|_{t=t_{1}} \leq\left.\mathrm{e}\left[g \mid g_{\infty}\right]\right|_{t=t_{0}} \mathrm{e}^{-\gamma t_{1}}
$$

so for any $t \in\left(t_{1}, t_{2}\right)$ we have

$$
\mathrm{e} \leq\left.\mathrm{e}\right|_{t=t_{1}} \mathrm{e}^{-\gamma\left(t-t_{1}\right)} \leq\left.\mathrm{e}\right|_{t=0} \mathrm{e}^{-\gamma t_{1}} \mathrm{e}^{-\gamma\left(t-t_{1}\right)}=\left.\mathrm{e}\right|_{t=0} \mathrm{e}^{-\gamma t},
$$

and so on for the subsequent intervals.

Corollary 2.4. Under the hypothesis of Theorem 2.2. $g_{\infty}$ also exponentially attracts $g$ in the sense of $L^{1}$ :

$$
\int_{S^{1}}\left|g(x, t)-g_{\infty}(x, t)\right| d x \leq C \mathrm{e}^{-\gamma t / 2}, t \geq 0
$$

where $C$ depends on $g$ and $g_{\infty}$.

Proof. It suffices to combine (2.15) with the Csiszár-Kullback inequality (2.12).

Returning to problem (2.1), put

$$
v_{\infty}=-\frac{1}{T} \int_{0}^{T} \int_{0}^{1} \Psi_{x}(x, t) g_{\infty}(x, t) d x d t
$$


where $g_{\infty}$ is the periodic solution of (2.7) with $F=-\Psi_{x}$ satisfying (2.11). Combining (2.10) and (2.18), we easily obtain the next result.

Corollary 2.5. Suppose that $\Psi(x, t)$ is $C^{4}$-smooth in $x$, and $F=-\Psi_{x}$ satisfies the hypothesis of Theorem 2.2. Then for any solution $\rho$ of (2.1) with the initial condition $\rho_{0}$ satisfying (2.2) we have

$$
\left|v_{\left[t_{0}, t_{0}+T\right]}-v_{\infty}\right| \leq C \mathrm{e}^{-\gamma t_{0} / 2},
$$

where $\gamma>0$ is the same as in Theorem 2.2 and depends only on $g_{\infty}$, and $C$ depends on $\rho$ and $g_{\infty}$.

We conclude that under the hypothesis of Corollary 2.5 the limiting average drift velocity is the same for all solutions of (2.1) and is determined by the periodic equation (2.7). For this reason in what follows we mostly concentrate on equation (2.7).

2.3. Tilting and tilted ratchets. An important class of unbiased potentials are the tilting potentials, which have the form

$$
\Psi(x, t)=\psi(x)+H(t) x,
$$

where the base potential $\psi(x)$ is 1 -periodic in $x$, and $H(t)$ is $T$-periodic in $t$ and characterized by the property

$$
\int_{0}^{T} H(t) d t=0
$$

Given the periodicity of $\psi$, equation (2.22) is equivalent to (2.3).

We obtain a typical tilting potential by letting

$$
H(t)=h(t) \omega,
$$

where $\omega \in \mathbb{R}$ characterizes the swing of the tilt, and

$$
h(t)=\left\{\begin{array}{ll}
1 & \text { if } k T<t \leq(k+1 / 2) T, \\
-1 & \text { if }(k+1 / 2) T<t \leq(k+1) T,
\end{array}(k=0,1, \ldots) .\right.
$$

The tilting potential corresponding to (2.24) periodically switches between the tilted potentials $\psi(x) \pm \omega x$. Thus the tilted potential

$$
\psi(x)+\omega x
$$

is a useful example of an obviously 'biased' potential (here $\psi$ satisfies the same conditions as above and $\omega$ is a number).

Remark 2.6. Observe that if $\Psi$ is a tilting ratchet potential (2.21), then

$$
v_{\left[t_{0}, t_{0}+T\right]}=-\frac{1}{T} \int_{t_{0}}^{t_{0}+T} \int_{0}^{1} \psi_{x}(x) g(x, t) d x d t .
$$


To prove this, substitute (2.21) in (2.10) and use the conservation of mass (2.11) and the zero mean condition (2.22). Consequently,

$$
v_{\infty}=-\frac{1}{T} \int_{0}^{T} \int_{0}^{1} \psi_{x}(x, t) g_{\infty}(x, t) d x d t .
$$

It is useful to consider the stationary periodic equation

$$
\sigma g_{x x}+\left(\left(\psi_{x}+\omega\right) g\right)_{x}=0, \quad(t, x) \in(0, \infty) \times S^{1},
$$

where $\psi_{x}$ is the derivative of a $C^{4} 1$-periodic function $\psi$. This equation can be treated by elementary methods. Put

$$
\begin{gathered}
\alpha=\alpha(\omega)=\mathrm{e}^{\omega}-1 ; \\
\beta_{+}=\beta_{+}(\omega, \psi)=\int_{0}^{1} \mathrm{e}^{\omega x+\psi(x)} d x ; \\
\beta_{-}=\beta_{-}(\omega, \psi)=\int_{0}^{1} \mathrm{e}^{-\omega x-\psi(x)} d x ; \\
\beta=\beta(\omega, \psi)=\int_{0}^{1} \int_{0}^{x} \mathrm{e}^{\omega y+\psi(y)-\omega x-\psi(x)} d y d x
\end{gathered}
$$

and define

$$
A(\omega, \psi)=\frac{\alpha}{\alpha \beta+\beta_{+} \beta_{-}}, \quad B(\omega, \psi)=\frac{\beta_{+}}{\alpha \beta+\beta_{+} \beta_{-}} .
$$

Remark 2.7. Observe that $A(\omega, \psi)>0$ for $\omega>0$ and $A(0, \psi)=0$. A simple reflection argument $(\psi(x) \mapsto \psi(1-x), \omega \mapsto-\omega)$ shows that $A(\omega, \psi)<0$ for $\omega<0$. In particular, $A(\omega, \psi)$ exists for any $\omega$ and continuous $\psi$ (i.e., the denominator does not vanish). Consequently, $B(\omega, \psi)$ also exists.

Proposition 2.8. Suppose $\psi: \mathbb{R} \rightarrow \mathbb{R}$ is continuously differentiable and 1-periodic; then (2.28) has a unique solution $g^{*}$ such that

$$
g^{*}(x) \geq 0, \quad \int_{0}^{1} g^{*}(x) d x=1 .
$$

Moreover, $g$ is given by

$$
g^{*}=\mathrm{e}^{-(\omega x+\psi(x)) / \sigma}\left(B\left(\frac{\omega}{\sigma}, \frac{\psi}{\sigma}\right)+A\left(\frac{\omega}{\sigma}, \frac{\psi}{\sigma}\right) \int_{0}^{x} \mathrm{e}^{(\omega y+\psi(y)) / \sigma}\right) .
$$

Proof. The proof is straightforward, cf. [5].

Observe that $g^{*}$ is the normalized time-periodic solution of (2.7) with $F=-\left(\psi_{x}+\omega\right)$. Applying Theorem 2.2 , we immediately obtain the following corollary. 
Corollary 2.9. If $g$ solves

$$
\left\{\begin{array}{l}
g_{t}-\sigma g_{x x}-\left(\left(\psi_{x}+\omega\right) g\right)_{x}=0, \quad x \in S^{1}, t>0, \\
g(x, 0)=g_{0}(x), \quad x \in S^{1},
\end{array}\right.
$$

where $g_{0} \in L^{1}\left(S^{1}\right)$ is as in (2.8), and $g_{0} \ln g_{0} \in L^{1}$, then

$$
\mathrm{e}\left[g(\cdot, t) \mid g^{*}\right] \leq \mathrm{e}\left[g(\cdot, 0) \mid g^{*}\right] \mathrm{e}^{-\gamma t}, \quad t \geq 0,
$$

where $\gamma$ only depends on the lower and upper bounds of $g^{*}$ and on $\sigma$.

Remark 2.10. The normalized periodic solution $g^{*}$ of (2.28) satisfies

$$
\sigma g_{x}^{*}+\left(\psi_{x}+\omega\right) g^{*}=\sigma A
$$

with $A=A(\omega / \sigma, \psi / \sigma)$. Integrating, we get

$$
\sigma A=\int_{0}^{1}\left(\psi_{x}+\omega\right) g^{*} d x
$$

Incidentally, we see that

$$
v_{\infty}=-\int_{0}^{1}\left(\psi_{x}+\omega\right) g^{*} d x=-\sigma A
$$

where $v_{\infty}$ is the asymptotic drift velocity for the tilted potential $\psi+\omega x$ (with arbitrary $T>0$ ).

\section{Adiabatic limit for tilting Ratchets}

3.1. Asymptotic speed. In this section we consider the tilting ratchet given by

$$
\left\{\begin{array}{l}
\rho_{t}-\rho_{x x}-\left(\left(\psi_{x}+h(t) \omega\right) \rho\right)_{x}=0, \quad x \in \mathbb{R}, t>0 \\
\rho=\rho_{0}(x), \quad x \in \mathbb{R} ; \quad \rho_{0}(x) \geq 0, \quad \int_{-\infty}^{\infty} \rho_{0}(x) d x=1,
\end{array}\right.
$$

where $h$ is defined by (2.24) and for convenience $\sigma=1$. We focus on the adiabatic limit of (3.1), i.e., on its behaviour when $T$, the period of the tilting, is large. In this case we allow the diffusion to fully take its effect. Thus the transport in the adiabatic limit can be said to be driven by diffusion. Generally, the two tilted potentials $\psi(x) \pm$ $\omega x$ corresponding to problem (3.1) are not symmetric and produce drift velocities of different absolute values. For this reason the limiting average drift velocity of the tilting ratchet is nonzero.

The following theorem gives an effective formula for the adiabatic drift velocity. Before we state it, we introduce some notations. Consider the corresponding periodic problem

$$
g_{t}-g_{x x}-\left(\left(\psi_{x}+h(t) \omega\right) g\right)_{x}=0 \quad x \in S^{1}, t>0 ; \quad \int_{S^{1}} g d x=1
$$


It switches between the modes

$$
g_{t}-g_{x x}-\left(\left(\psi_{x}+\omega\right) g\right)_{x}=0 \quad x \in S^{1} ; \quad \int_{S^{1}} g d x=1
$$

and

$$
g_{t}-g_{x x}-\left(\left(\psi_{x}-\omega\right) g\right)_{x}=0 \quad x \in S^{1} ; \quad \int_{S^{1}} g d x=1
$$

spending a long time in each of them.

Let $g_{+}$and $g_{-}$be the stationary solutions of (3.3) and (3.4) respectively. By $\gamma_{+}$and $\gamma_{-}$denote the inverses of the Log-Sobolev constants (see (2.14) ) for the relative entropies

$$
\Sigma_{+}[g]=\mathrm{e}\left[g \mid g_{+}\right], \quad \Sigma_{-}[g]=\mathrm{e}\left[g \mid g_{-}\right]
$$

so by Corollary 2.9 for solutions of (3.3) we have the entropy decay

$$
\Sigma_{+}[g(\cdot, t)] \leq \Sigma_{+}\left[g\left(\cdot, t_{0}\right)\right] \mathrm{e}^{-\gamma_{+}\left(t-t_{0}\right)} \quad\left(t \geq t_{0}\right),
$$

and similarly for solutions of (3.4) we have

$$
\Sigma_{-}[g(\cdot, t)] \leq \Sigma_{-}\left[g\left(\cdot, t_{0}\right)\right] \mathrm{e}^{-\gamma_{-}\left(t-t_{0}\right)} \quad\left(t \geq t_{0}\right) .
$$

Let $g_{\infty}$ be the time-periodic solution of (3.2) with period $T$ and let $A(\omega)=A(\omega, \psi)$ and $A(-\omega)=A(-\omega, \psi)$ be defined according to (2.29). As $g_{\infty}$ is time-periodic, the asymptotic drift velocity of (3.1) can be expressed as

$$
v_{\infty}(T)=-\frac{1}{T} \int_{0}^{T} \int_{0}^{1} \psi_{x}(x) g_{\infty}(x, t) d x d t
$$

(see (2.27) ). Finally, put

$$
v_{\infty \infty}=-\frac{A(\omega)+A(-\omega)}{2}=-\frac{1}{2} \int_{0}^{1} \psi_{x}(x)\left(g_{+}(x)+g_{-}(x)\right) d x
$$

where the last equality is due to (2.32).

Theorem 3.1. Suppose $\psi \in C^{4}(\mathbb{R})$ is 1-periodic; then

$$
\left|v_{\infty}(T)-v_{\infty \infty}\right| \leq \frac{2^{3 / 2}}{T} \max \left|\psi_{x}(x)\right|\left(\frac{\sqrt{\Sigma_{+}\left[g_{-}\right]}}{\gamma_{+}}+\frac{\sqrt{\Sigma_{-}\left[g_{+}\right]}}{\gamma_{-}}\right)+o\left(\frac{1}{T}\right) .
$$

In particular, if $v_{\infty \infty} \neq 0$, for large $T$ there is nonzero unidirectional transport.

Before we prove Theorem 3.1 we must obtain some auxiliary results. Put

$$
M_{+}=\max \left|\ln \frac{g_{-}}{g_{+}}\right| \sqrt{2}, \quad M_{-}=\max \left|\ln \frac{g_{+}}{g_{-}}\right| \sqrt{2}
$$


and consider the functions

$$
\phi_{+}(R)=R+M_{+} \sqrt{R}, \quad \phi_{-}(R)=R+M_{-} \sqrt{R} .
$$

Observe that $\phi_{+}$and $\phi_{-}$are continuous and increasing on $\mathbb{R}_{+}$.

Lemma 3.2. For any probability density $g \in L^{1}\left(S^{1}\right)$ such that $g \ln g \in$ $L^{1}\left(S^{1}\right)$, we have

$$
\begin{aligned}
& \left|\Sigma_{+}[g]-\Sigma_{+}\left[g_{-}\right]\right| \leq \phi_{+}\left(\Sigma_{-}[g]\right), \\
& \left|\Sigma_{-}[g]-\Sigma_{-}\left[g_{+}\right]\right| \leq \phi_{-}\left(\Sigma_{+}[g]\right) .
\end{aligned}
$$

Proof. A straightforward computation yields

$$
\begin{aligned}
& \Sigma_{+}[g]-\Sigma_{+}\left[g_{-}\right]=\int_{S^{1}} g \ln \frac{g}{g_{+}} d x-\int_{S^{1}} g_{-} \ln \frac{g_{-}}{g_{+}} d x \\
&=\int_{S^{1}}\left(g \ln \frac{g}{g_{-}}+\left(g-g_{-}\right) \ln \frac{g_{-}}{g_{+}}\right) d x
\end{aligned}
$$

whence

$$
\left|\Sigma_{+}[g]-\Sigma_{+}\left[g_{-}\right]\right| \leq \Sigma_{-}[g]+\frac{M_{+}}{\sqrt{2}}\left\|g-g_{-}\right\|_{L^{1}\left(S^{1}\right)} .
$$

Now it remains to apply the Csiszár-Kullback inequality (2.12) and obtain (3.10).

Inequality (3.11) is proved by swapping $g_{+}$and $g_{-}$.

Note that the periodic solution $g_{\infty}$ of $(3.2)$ implicitly depends on $T$, which is a parameter of tilting.

Lemma 3.3. We have

$$
\lim _{T \rightarrow \infty} \Sigma_{+}\left[g_{\infty}(\cdot, 0)\right]=\Sigma_{+}\left[g_{-}\right] ; \quad \lim _{T \rightarrow \infty} \Sigma_{-}\left[g_{\infty}(\cdot, T / 2)\right]=\Sigma_{-}\left[g_{+}\right] .
$$

Proof. We only prove the first limit in (3.12), as the proof of the second one is completely analogous.

Take an arbitrary $R>0$ and for any $\varepsilon>0$ choose $T_{\varepsilon}>0$ in such a way that

$$
\begin{gathered}
\left(\Sigma_{+}\left[g_{-}\right]+\varepsilon\right) \mathrm{e}^{-\gamma_{+} T_{\varepsilon} / 2} \leq R, \\
\left(\Sigma_{-}\left[g_{+}\right]+\phi_{-}(R)\right) \mathrm{e}^{-\gamma_{-} T_{\varepsilon} / 2} \leq \phi_{+}^{-1}(\varepsilon) .
\end{gathered}
$$

Now take $T>T_{\varepsilon}$ and let $\mathcal{T}$ be the resolving operator for (3.2) taking $g(\cdot, 0)$ to $g(\cdot, T)$. We claim that $\mathcal{T}$ maps the set $X=\left\{g_{0} \in W_{2}^{1}\left(S^{1}\right) \cap\{\right.$ probability densities $\left.\}|| \Sigma_{+}\left[g_{0}\right]-\Sigma_{+}\left[g_{-}\right] \mid \leq \varepsilon\right\}$ 
into itself. To prove this, consider a solution $g$ of (3.2) with the initial condition $g(\cdot, 0)=g_{0} \in X$. Thanks to the conservation of mass, it only remains to prove that

$$
\left|\Sigma_{+}[g(\cdot, T)]-\Sigma_{+}\left[g_{-}\right]\right| \leq \varepsilon .
$$

Indeed, making use of (3.10), (3.11), and the attraction (3.5) and (3.6) we consequently obtain

$$
\begin{gathered}
\Sigma_{+}[g(\cdot, 0)]=\Sigma_{+}\left[g_{0}\right] \leq \Sigma_{+}\left[g_{-}\right]+\varepsilon ; \\
\Sigma_{+}[g(\cdot, T / 2)] \leq \Sigma_{+}\left[g_{0}\right] \mathrm{e}^{-\gamma_{+} T / 2} \leq\left(\Sigma_{+}\left[g_{-}\right]+\varepsilon\right) \mathrm{e}^{-\gamma_{+} T / 2} \leq R ; \\
\Sigma_{-}[g(\cdot, T / 2)] \leq \Sigma_{-}\left[g_{+}\right]+\phi_{-}\left(\Sigma_{+}[g(\cdot, T / 2)]\right) \leq \Sigma_{-}\left[g_{+}\right]+\phi_{-}(R) ; \\
\Sigma_{-}[g(\cdot, T)] \leq \Sigma[g(\cdot, T / 2)] \mathrm{e}^{-\gamma-T / 2} \leq\left(\Sigma_{-}\left[g_{+}\right]+\phi_{-}(R)\right) \mathrm{e}^{-\gamma_{-} T / 2} \leq \phi_{+}^{-1}(\varepsilon) ; \\
\left|\Sigma_{+}[g(\cdot, T)]-\Sigma_{+}\left[g_{-}\right]\right| \leq \phi_{+}\left(\Sigma_{-}[g(\cdot, T)]\right) \leq \phi_{+}\left(\phi_{+}^{-1}(\varepsilon)\right)=\varepsilon,
\end{gathered}
$$

so (3.13) holds, and $X$ is invariant under $\mathcal{T}$. Moreover, $X$ is closed in $W_{2}^{1}\left(S^{1}\right)$, convex, bounded in $L^{1}\left(S^{1}\right)$, and by parabolic regularity $\mathcal{T}: X \rightarrow X$ is continuous and $\mathcal{T}(X)$ is precompact in $W_{2}^{1}\left(S^{1}\right)$. By the Schauder fixed point theorem $\mathcal{T}$ has a fixed point in $X$, which is the initial data for a time-periodic solution of (3.2). Due to uniqueness of such a periodic solution, this fixed point coincides with $g_{\infty}(\cdot, 0)$. This implies that $\left|\Sigma_{+}\left[g_{\infty}(\cdot, 0)\right]-\Sigma_{+}\left[g_{-}\right]\right| \leq \varepsilon$ whenever $T \geq T_{\varepsilon}$, and the first limit in (3.12) is proved.

Proof of Theorem 3.1. Using (3.7) and (3.8), we estimate the difference on the left-hand side of (3.9) as follows:

$$
\begin{aligned}
& \mid v_{\infty}(T)- v_{\infty \infty} \mid \\
&=\left|-\frac{1}{T} \int_{0}^{T} \int_{0}^{1} \psi_{x} g_{\infty} d x d t+\frac{1}{2} \int_{0}^{1} \psi_{x}\left(g_{+}+g_{-}\right) d x\right| \\
&= \mid-\frac{1}{T} \int_{0}^{T / 2} \int_{0}^{1} \psi_{x} g_{\infty} d x d t-\frac{1}{T} \int_{T / 2}^{T} \int_{0}^{1} \psi_{x} g_{\infty} d x d t \\
&+\frac{1}{T} \int_{0}^{T / 2} \int_{0}^{1} \psi_{x} g_{+} d x d t+\frac{1}{T} \int_{T / 2}^{T} \int_{0}^{1} \psi_{x} g_{-} d x d t \mid \\
& \leq \frac{1}{T} \int_{0}^{T / 2} \int_{0}^{1}\left|\psi_{x}\left(g_{\infty}-g_{+}\right)\right| d x d t+\frac{1}{T} \int_{T / 2}^{T} \int_{0}^{1}\left|\psi_{x}\left(g_{\infty}-g_{-}\right)\right| d x d t \\
& \leq \frac{1}{T} \max _{x \in[0,1]}\left|\psi_{x}(x)\right|\left(\int_{0}^{T / 2}\left\|g_{\infty}-g_{+}\right\|_{L^{1}\left(S^{1}\right)} d t+\int_{T / 2}^{T}\left\|g_{\infty}-g_{-}\right\|_{L^{1}\left(S^{1}\right)} d t\right)
\end{aligned}
$$


Applying the Csiszár-Kullback inequality, we obtain

$$
\begin{aligned}
& \left|v_{\infty}(T)-v_{\infty \infty}\right| \\
& \leq \frac{\sqrt{2}}{T} \max _{x \in[0,1]}\left|\psi_{x}(x)\right|\left(\int_{0}^{T / 2} \sqrt{\Sigma_{+}\left[g_{\infty}\right]} d t+\int_{T / 2}^{T} \sqrt{\Sigma_{-}\left[g_{\infty}\right]} d t\right) .
\end{aligned}
$$

As $g_{\infty}(x, t)$ solves (3.3) for $t \in[0, T / 2)$ and (3.4) for $t \in[T / 2, T)$, we can apply the entropy attraction (3.5) and (3.6) and obtain

$$
\begin{aligned}
& \left|v_{\infty}(T)-v_{\infty \infty}\right| \\
& \leq \frac{\sqrt{2}}{T} \max _{x \in[0,1]}\left|\psi_{x}(x)\right|\left(\sqrt{\Sigma_{+}\left[g_{\infty}(\cdot, 0)\right]} \int_{0}^{T / 2} \mathrm{e}^{-\gamma_{+} t / 2} d t\right. \\
& \left.\quad+\sqrt{\Sigma_{-}\left[g_{\infty}(\cdot, T / 2)\right]} \int_{T / 2}^{T} \mathrm{e}^{-\gamma_{-}(t-T / 2) / 2} d t\right) \\
& \leq \frac{2^{3 / 2}}{T} \max _{x \in[0,1]}\left|\psi_{x}(x)\right|\left(\frac{1}{\gamma_{+}} \sqrt{\Sigma_{+}\left[g_{\infty}(\cdot, 0)\right]}+\frac{1}{\gamma_{-}} \sqrt{\Sigma_{-}\left[g_{\infty}(\cdot, T / 2)\right]}\right) .
\end{aligned}
$$

The last estimate and the limits (3.12) yield (3.9).

Remark 3.4. The proof of Lemma 3.3 gives opportunity to estimate the term $o(1 / T)$ on the right-hand side of (3.9).

Remark 3.5. If $\psi$ is fixed, $v_{\infty \infty}=-(A(\omega)+A(-\omega)) / 2$ is an analytic function of $\omega$. Consequently, it either identically equals 0 or has at most countably many zeroes without accumulation points.

Remark 3.6. Having in mind (2.33), we see that the adiabatic drift velocity $v_{\infty \infty}$ equals the arithmetic mean of the limiting drift velocities for the tilted potentials $\psi(x) \pm \omega x$.

Remark 3.7. Observe that given $\psi$ and $\omega$, it is trivial to compute $A(\omega)$ numerically. In this sense Theorem 3.1 is effective.

3.2. Bulk transport direction. There is another formula that allows one to determine the direction of the adiabatic transport. Put

$$
J=J(\psi, \omega)=\frac{2 \int_{0}^{1} \int_{0}^{x} \sinh (\psi(x)-\psi(y)) \sinh [\omega(x-y-1 / 2)] d y d x}{\sinh (\omega / 2)}
$$

Proposition 3.8. If $\omega \neq 0$, the sign of $v_{\infty \infty}$ coincides with the sign of $J$. Consequently, if $J>0(J<0)$, then the adiabatic transport goes in the positive (respectively, negative) direction. 
Proof. Using the definition of $A$ (2.29), write

$$
\begin{aligned}
\frac{1}{A(\omega)}=\frac{1}{\mathrm{e}^{\omega}-1}( & \left(\mathrm{e}^{\omega}-1\right) \int_{0}^{1} \int_{0}^{x} \mathrm{e}^{\psi(y)-\psi(x)+\omega(y-x)} d y d x \\
& \left.+\int_{0}^{1} \int_{0}^{1} \mathrm{e}^{\psi(y)-\psi(x)+\omega(y-x)} d y d x\right) \\
= & \frac{1}{\mathrm{e}^{\omega}-1}\left(\mathrm{e}^{\omega} \int_{0}^{1} \int_{0}^{x} \mathrm{e}^{\psi(y)-\psi(x)+\omega(y-x)} d y d x\right. \\
& \left.+\int_{0}^{1} \int_{x}^{1} \mathrm{e}^{\psi(y)-\psi(x)+\omega(y-x)} d y d x\right) \\
= & \frac{\mathrm{e}^{\omega / 2}}{\mathrm{e}^{\omega}-1} \int_{0}^{1} \int_{0}^{x}\left(\mathrm{e}^{\psi(y)-\psi(x)+\omega(y-x+1 / 2)}\right. \\
& \left.+\mathrm{e}^{-(\psi(y)-\psi(x)+\omega(y-x+1 / 2))} d y d x\right) \\
=\frac{1}{\sinh (\omega / 2)} & \int_{0}^{1} \int_{0}^{x} \cosh (\psi(y)-\psi(x)+\omega(y-x+1 / 2)) d y d x
\end{aligned}
$$

Substituting $-\omega$ for $\omega$, we obtain

$$
\frac{1}{A(-\omega)}=-\frac{\int_{0}^{1} \int_{0}^{x} \cosh (\psi(y)-\psi(x)-\omega(y-x+1 / 2)) d y d x}{\sinh (\omega / 2)} .
$$

Summing and converting the difference of hyperbolic cosines into product, we get

$$
\frac{1}{A(\omega)}+\frac{1}{A(-\omega)}=J
$$

Now it suffices to observe that as $A(\omega)$ and $A(-\omega)$ have opposite signs, so do the sums $A(\omega)+A(-\omega)=-2 v_{\infty \infty}$ and $1 / A(\omega)+1 / A(-\omega)=$ $J$.

Example 3.9. Symmetric potentials satisfying $\psi(x)=\psi(1-x)$ do not produce adiabatic transport. This follows e.g. from Proposition 3.8. Indeed, if $\psi$ is symmetric, by changing the variables $x^{\prime}=1-y, y^{\prime}=1-x$ in (3.14) we get $J(\psi, \omega)=-J(\psi, \omega)$, whence $J=0$. Note however, that symmetric potentials can produce transport if the tilting regime is asymmetric in time unlike (2.24), see below.

Example 3.10. Supersymmetric potentials (see [32]) satisfying $-\psi(x)=$ $\psi(x+1 / 2)$ do not produce adiabatic transport either. This, too, can be derived from Proposition 3.8. Indeed, utilizing in (3.14) the change 
of variables $x^{\prime}=y+1 / 2, y^{\prime}=x-1 / 2$ on the set

$$
Q=\left\{(x, y): \frac{1}{2} \leq x \leq 1,0 \leq y \leq \frac{1}{2}\right\},
$$

and the change of variables $x^{\prime \prime}=x+1 / 2, y^{\prime \prime}=y+1 / 2$ on the rest of the triangle

$$
\{(x, y): 0 \leq x \leq 1,0 \leq y \leq x\} \backslash Q
$$

we deduce that $J=0$.

Example 3.11. Simple examples of asymmetric potentials such as $\psi(x)=\cos \left(2 \pi x^{m}\right)$ suggest that if $\psi$ increases (decreases) on a major interval, then the direction of adiabatic transport is positive (resp. negative). The following theorem justifies this claim.

Theorem 3.12. Suppose that $\psi \in C^{4}\left(S^{1}\right)$ strictly increases along an oriented arc $\overrightarrow{[\alpha, \beta]}$. Let $h: S^{1} \times[0,1] \rightarrow S^{1}$ be a homotopy such that

(1) $h(\cdot, 0)$ is the identity mapping on $S^{1}$;

(2) for any $\lambda \in[0,1)$ the mapping $h(\cdot, \lambda): S^{1} \rightarrow S^{1}$ is $C^{4}$;

(3) $h(\cdot, 1)$ preserves the orientation on the oriented arc $\overrightarrow{[\alpha, \beta]}$;

(4) $h(\cdot, 1)$ maps the oriented arc $\overrightarrow{[\beta, \alpha]}$ onto a single point.

Let $v_{\infty \infty}(\lambda)$ be the asymptotic velocity corresponding to $\psi_{\lambda}:=$ $\psi(h(\cdot, \lambda))$, where $0 \leq \lambda<1$. Then $v_{\infty \infty}(\lambda)>0$ if $\lambda$ is sufficiently close to 1 .

Proof. Without loss of generality, $\omega>0$ and $\alpha=0=h(\alpha, \lambda)$ for $\lambda \in[0,1]$. We use Proposition [3.8. Since for any $x \in S^{1}$ we have $\psi_{\lambda}(x) \rightarrow \psi_{1}(x)$ as $\lambda \rightarrow 1$, by the Lebesgue dominated convergence theorem we obtain

$$
=\frac{2}{\sinh (\omega / 2)} \int_{0}^{1} \int_{0}^{x} \sinh \left(\psi_{1}(x)-\psi_{1}(y)\right) \sinh \omega(x-y-1 / 2) d y d x .
$$

The homotopy $h$ maps the interior of the arc $\overrightarrow{[\alpha, \beta]}$ onto $S^{1} \backslash\{0\}$ and preserves the orientation, so $\psi_{1}$ increases on the interval $(0,1)$. Now the right-hand side of (3.15) is positive by Proposition 3.14 (see below). Consequently, if $\lambda$ is sufficiently close to $1, J\left(\omega, \psi_{\lambda}\right)$ is also positive and so is $v_{\infty \infty}(\lambda)$.

Remark 3.13. In the notations of Theorem 3.12 the exact formula for the limiting velocity is

$$
\lim _{\lambda \rightarrow 1} v_{\infty \infty}(\lambda)=-\frac{A\left(\omega, \psi_{1}\right)+A\left(-\omega, \psi_{1}\right)}{2} .
$$


It does not depend on the values of the initial potential $\psi$ on the arc $\overrightarrow{[\beta, \alpha]}$ that collapses to a point.

It remains to prove the following functional inequality.

Proposition 3.14. Let $f:[0,1] \rightarrow \mathbb{R}$ be any increasing continuous function, $\varphi:[0,+\infty) \rightarrow \mathbb{R}$ be a convex $C^{1}$-smooth function, and $\Phi:$ $\mathbb{R} \rightarrow \mathbb{R}$ be an odd continuous function such that $x>0$ implies $\Phi(x)>0$. Then

$$
\int_{0}^{1} \int_{0}^{x} \varphi(f(x)-f(y)) \Phi(x-y-1 / 2) d y d x \geq 0 .
$$

Moreover, if $f$ is strictly increasing and $\varphi$ is strictly convex (in the sense that $\varphi^{\prime}$ is strictly increasing), then inequality (3.17) is strict.

We first prove two auxiliary statements.

Lemma 3.15. Let $\varphi$ be as above, and $x_{1}, \ldots, x_{2 m}, m \in \mathbb{N}$, be a collection of non-negative numbers. Then

$$
\sum_{i=1}^{m} \varphi\left(\sum_{j=1}^{m+1} x_{i+j-1}\right) \geq \sum_{i=1}^{m+1} \varphi\left(\sum_{j=1}^{m} x_{i+j-1}\right) .
$$

If $\varphi$ is strictly convex and the numbers $x_{1}, \ldots, x_{2 m}$ are positive, then this inequality is strict.

Proof. It suffices to observe that all the partial derivatives of the function $F\left(x_{1}, \ldots, x_{2 m}\right)=\sum_{i=1}^{m} \varphi\left(\sum_{j=1}^{m+1} x_{i+j-1}\right)-\sum_{i=1}^{m+1} \varphi\left(\sum_{j=1}^{m} x_{i+j-1}\right)$ are non-negative (and even positive provided $\varphi$ is strictly convex and $x_{1}, \ldots, x_{2 m}$ are positive), and $F(0, \ldots, 0)=0$.

Lemma 3.16. Let $\varphi$ and $f$ be as above, and $a<b$ be two positive numbers. Then

$$
\int_{0}^{a} \varphi(f(x+b)-f(x)) d x \geq \int_{0}^{b} \varphi(f(x+a)-f(x)) d x .
$$

If $f$ is strictly increasing, $\varphi$ is strictly convex, and $a / b$ is a rational number, then inequality (3.19) is strict.

Proof. Assume first that the ratio $a / b$ is equal to $m / m+1$ with some natural number $m$. After rescaling, without loss of generality we may 
suppose that $a=m$. In this case, inequality (3.19) may be rewritten as

$$
\begin{aligned}
\sum_{i=1}^{m} \int_{0}^{1} \varphi(f(x+i+m)-f(x+i-1)) d x & \\
& \geq \sum_{i=1}^{m+1} \int_{0}^{1} \varphi(f(x+i+m-1)-f(x+i-1)) d x,
\end{aligned}
$$

or

$$
\begin{aligned}
& \sum_{i=1}^{m} \int_{0}^{1} \varphi\left(\sum_{j=1}^{m+1} f(x+i+j-1)-f(x+i+j-2)\right) d x \\
& \quad \geq \sum_{i=1}^{m+1} \int_{0}^{1} \varphi\left(\sum_{j=1}^{m} f(x+i+j-1)-f(x+i+j-2)\right) d x
\end{aligned}
$$

and thus the statement of the lemma follows from Lemma 3.15.

If $a / b$ is a rational number $\frac{m}{n}$, with $m, n \in \mathbb{N}, m<n$, then it can be decomposed as $\frac{m}{m+1} \cdot \frac{m+1}{m+2} \cdot \cdots \cdot \frac{n-1}{n}$, and the statement of the lemma follows from the previous case. By continuity, the non-strict inequality (3.19) holds for all irrational $a / b$.

Proof of Proposition 3.14. We make a change of variables in the double integral (3.17), and conclude that it is equal to

$$
\begin{aligned}
& \int_{-1 / 2}^{1 / 2} \int_{0}^{1 / 2-\xi} \varphi(f(y+\xi+1 / 2)-f(y)) \Phi(\xi) d y d \xi \\
& \quad=\int_{0}^{1 / 2}\left(\int_{0}^{1 / 2-\xi} \varphi(f(y+\xi+1 / 2)-f(y)) d y\right. \\
& \left.\quad-\int_{0}^{1 / 2+\xi} \varphi(f(y-\xi+1 / 2)-f(y)) d y\right) \Phi(\xi) d \xi \geq 0
\end{aligned}
$$


by Lemma 3.16. When $f$ is strictly increasing and $\varphi$ is strictly convex, this integral is strictly positive since the set

$$
\begin{aligned}
\left\{\xi \in\left(0, \frac{1}{2}\right) \mid \int_{0}^{1 / 2-\xi} \varphi(f(y+\xi+1 / 2)-f(y)) d y\right. & \\
> & \left.\int_{0}^{1 / 2+\xi} \varphi(f(y-\xi+1 / 2)-f(y)) d y\right\}
\end{aligned}
$$

is open and non-empty (since it contains the rational numbers), and therefore has non-zero Lebesgue measure.

\section{Semiadiabatic limit and the stochastic Stokes' Drift}

Consider the tilting ratchet with the potential

$$
\Psi(x, t)=\psi(x)+H(t) x,
$$

where

$$
H(t)= \begin{cases}\Omega, & 0<t<\tau, \\ \omega, & \tau<t<T,\end{cases}
$$

and as usual $\psi(x)$ is 1-periodic in $x$ and $H(t)$ is $T$-periodic in $T$. We assume that the non-bias condition (2.22) is satisfied, i.e.,

$$
\Omega \tau+\omega(T-\tau)=0 .
$$

We regard $T, \tau$, and $\omega$ as the independent parameters of the tilting ratchet; $\Omega$ can be expressed via the independent parameters by means of (4.3).

We study the regime (4.1) in the so-called semiadiabatic limit supposing that $T$ is large, $\tau / T$ is small, and $\omega$ is constant. It follows from (4.3) that in the semiadiabatic limit $|\Omega|$ is large.

As before, let $v_{\infty}=v_{\infty}(\omega, T, \tau)$ denote the eventual drift velocity given by (2.19). Further, let $g^{*}$ be the 1-periodic solution of the problem

$$
g_{x x}+\left(\left(\psi_{x}+\omega\right) g\right)_{x}=0, \quad \int_{0}^{1} g(x) d x=1,
$$

which also satisfies

$$
g_{x}^{*}+\left(\psi_{x}+\omega\right) g^{*}=A(\omega)
$$

with $A(\omega)$ defined by (2.29) (see Remark 2.10). Put

$$
v_{\infty \infty}(\omega)=-\int_{0}^{1} \psi_{x} g^{*} d x \equiv \omega-A(\omega) .
$$


Our main result concerning the semiadiabatic tilting is that $v_{\infty \infty}(\omega)$ is the asymptotic average drift velocity as $T \rightarrow \infty$ and $\tau / T \rightarrow 0$, and its sign coincides with the sign of $\omega$ provided that $\psi$ is nontrivial. For definitiveness we assume that $\omega>0$.

First we address the positivity of $v_{\infty \infty}$. It will follow from the next proposition.

Proposition 4.1. Given $\omega>0$, the functional

$$
\mathcal{V}_{\omega}(F)=\alpha(\omega) \int_{0}^{1} \int_{0}^{x} \frac{F(y)}{F(x)} d y d x+\int_{0}^{1} \int_{0}^{1} \frac{F(y)}{F(x)} d y d x,
$$

where $F \in C[0,1], F(x)>0$ for all $x \in[0,1]$, attains its global minimum $\alpha(\omega) / \omega$ when and only when $F(x)=C \mathrm{e}^{\omega x}$.

Proof. Since the functional is homogeneous of degree zero, it suffices to prove that under the additional restriction

$$
\int_{0}^{1} F(y) d y=1
$$

the only minimizer is $F(x)=\frac{\omega}{\alpha(\omega)} \mathrm{e}^{\omega x}$.

Let $G(x)=1+\alpha(\omega) \int_{0}^{x} F(y) d y$. Then $G(0)=1, G(1)=\mathrm{e}^{\omega}, G^{\prime}(\cdot)>0$, and our functional becomes

$$
\tilde{\mathcal{V}}_{\omega}(G)=\alpha(\omega) \int_{0}^{1} \frac{G(x)}{G^{\prime}(x)} d x .
$$

Let now $H(x)=\ln G(x)$. Then $H(0)=0, H(1)=\omega, H^{\prime}(\cdot)>0$, and

$$
\tilde{\tilde{\mathcal{V}}}_{\omega}(H)=\alpha(\omega) \int_{0}^{1} \frac{1}{H^{\prime}(x)} d x .
$$

The Cauchy-Bunyakovskii-Schwarz inequality implies that

$$
\int_{0}^{1} \frac{1}{H^{\prime}(x)} d x \geq \frac{1}{\int_{0}^{1} H^{\prime}(x) d x}=\frac{1}{\omega},
$$

and the equality holds only if $H^{\prime}(x) \equiv \omega$. This means that the minimum of $\mathcal{V}_{\omega}$ is achieved merely if $H(x)=\omega x, G(x)=\mathrm{e}^{\omega x}$ and $F(x)=\frac{\omega}{\alpha(\omega)} \mathrm{e}^{\omega x}$. 
Remark 4.2. It is interesting to observe that the functionals (4.6) and (4.8) neither are convex nor fit into the framework of general $L^{1}$-lower semicontinuity criteria which go back to [35] (see e.g. [16] for a review).

Proposition 4.3. Given a 1-periodic $\psi \in C^{4}(\mathbb{R})$ and $\omega>0$, $v_{\infty \infty}$ satisfies

$$
0 \leq v_{\infty \infty} \equiv \omega-A(\omega)<\omega .
$$

Moreover, $v_{\infty \infty}=0$ if and only if $\psi$ is constant.

Proof. Elementary properties of $A$ (see Remark 2.7) yield the upper bound in (4.11). The lower bound is a corollary of Proposition 4.1. Indeed, put $F(x)=\mathrm{e}^{\psi(x)+\omega x}$ and write

$$
A=\frac{\alpha(\omega)}{\mathcal{V}_{\omega}(F)},
$$

where $\mathcal{V}_{\omega}$ is given by (4.6). According to Proposition 4.1, we have

$$
A \leq \frac{\alpha(\omega)}{\alpha(\omega) / \omega}=\omega
$$

and the lower bound in (4.11) is proved. Moreover, the bound is attained if and only if $F(x)=C \mathrm{e}^{\omega x}$, with some constant $C>0$, i.e., if and only if $\psi(x) \equiv \ln C$ is constant.

Write the Log-Sobolev inequality associated with $g^{*}$ in the form

$$
\int_{S^{1}} g \ln \frac{g}{g^{*}} d x \leq \frac{1}{\gamma} \int_{S^{1}} g\left|\left(\ln \frac{g}{g^{*}}\right)_{x}\right|^{2} d x .
$$

Put

$$
M_{0}=\max _{x \in[0,1]}\left|\psi_{x}(x)\right|, \quad M_{1}=\max _{x \in[0,1]}\left|\frac{g_{x}^{*}(x)}{g^{*}(x)}\right| .
$$

The following statements characterize the semiadiabatic limit of the tilting ratchets.

Theorem 4.4. Suppose that $\psi \in C^{4}(\mathbb{R})$ is 1-periodic and $\omega>0$, $0<\tau<T$; then the average drift velocity $v_{\infty}(\omega, T, \tau)$ satisfies

$$
\begin{aligned}
\mid v_{\infty}(\omega, T, \tau) & -v_{\infty \infty}(\omega) \mid \leq\left(M_{0}+v_{\infty \infty}(\omega)\right) \frac{\tau}{T} \\
+ & \frac{2^{3 / 2} M_{0} M_{1}^{1 / 2}}{\gamma} \omega^{1 / 2} \frac{1}{T^{1 / 2}}\left(1+\frac{1}{\mathrm{e}^{\gamma T(1-\tau / T)}-1}\right)^{1 / 2} .
\end{aligned}
$$

The proof will be given later in this section. 
Corollary 4.5. Under the hypothesis of Theorem 4.4, for any $\omega>0$, we have

$$
\lim _{\substack{T \rightarrow \infty \\ \tau / T \rightarrow 0}} v_{\infty}(\omega, T, \tau)=v_{\infty \infty}(\omega),
$$

the limit is uniform in $\omega \in\left(0, \omega_{0}\right]$ for any $\omega_{0}>0$, and the rate of convergence is $O\left(T^{-1 / 2}+\tau / T\right)$.

Proof. It suffices to observe that by Proposition 4.3 we have $v_{\infty \infty}(\omega) \leq$ $\omega$ and that

$$
1+\frac{1}{\mathrm{e}^{\gamma T(1-\tau / T)}-1}
$$

remains bounded as $T \rightarrow \infty$ and $\tau / T \rightarrow 0$.

Combining Corollary 4.5 and Proposition 4.3, we get the following corollary.

Corollary 4.6. For any non-constant 1-periodic $\psi \in C^{4}$ and any $\omega>$ 0 , we have $v_{\infty \infty}(\omega)>0$, if $T$ is sufficiently large and $\tau / T$ is sufficiently small.

In other words, Corollary 4.6 means that the semiadiabatic transport goes in the positive/negative direction according to the sign of $\omega$.

Before proving Theorem 4.4, we introduce some notations and prove a lemma. Denote by

$$
\Sigma[g]=\mathrm{e}\left[g \mid g^{*}\right]
$$

the relative entropy with respect to $g^{*}$, and let

$$
\mathrm{I}[g]=\int_{S^{1}} g\left|\left(\ln \frac{g}{g^{*}}\right)_{x}\right|^{2} d x
$$

be the corresponding entropy production term, then the Log-Sobolev inequality (4.12) can be written as

$$
\Sigma[g] \leq \frac{1}{\gamma} \mathrm{I}[g]
$$

In the case of semiadiabatic tilting, equation (2.7) splits into

$$
\begin{array}{ll}
g_{t}-g_{x x}-\left(\left(\psi_{x}+\Omega\right) g\right)_{x}=0, & (t, x) \in(0, \tau) \times S^{1} \\
g_{t}-g_{x x}-\left(\left(\psi_{x}+\omega\right) g\right)_{x}=0, & (t, x) \in(\tau, T) \times S^{1} .
\end{array}
$$

We consider (4.18)-(4.19) in the class

$$
g \geq 0 ; \quad \int_{S^{1}} g(x, t) d x=1 .
$$


As usual, $g_{\infty}$ denotes the unique $T$-periodic in $t$ solution of (4.18)(4.20), existing due to Theorem [2.2. Note that the function $g_{\infty}$ itself implicitly depends on the parameters of tilting $T, \tau$, and $\omega$.

Lemma 4.7. We have

$$
\Sigma\left[g_{\infty}(\cdot, \tau)\right] \leq M_{1} \omega T\left(1+\frac{1}{\mathrm{e}^{\gamma T(1-\tau / T)}-1}\right) .
$$

Proof. We start with an a priori estimate for a solution $g$ of (4.18)(4.20). First we estimate the entropy for $t \in[0, \tau]$. Put $r=g / g^{*}$, then $r_{t}=g_{t} / g^{*}$, and $(\ln r)_{t}=g_{t} / g$. We have:

$$
\frac{d}{d t} \Sigma[g]=\frac{d}{d t} \int_{S^{1}} g \ln r d x=\int_{S^{1}} g_{t} \ln r d x+\int_{S^{1}} g_{t} d x .
$$

Plugging in (4.18), using the conservation of mass (4.20) and integrating by parts, we proceed as follows:

$$
\begin{aligned}
\frac{d}{d t} \Sigma[g]=\int_{S^{1}}\left(g_{x x}+\left(\left(\psi_{x}+\Omega\right) g\right)_{x}\right) & \ln r d x \\
=- & \int_{S^{1}} g \frac{r_{x}^{2}}{r^{2}} d x+\int_{S^{1}} \frac{g}{r}\left(r_{x x}-\left(\psi_{x}+\Omega\right) r_{x}\right) d x \\
=-I[g]+\int_{S^{1}} g^{*}\left(r_{x x}-\left(\psi_{x}+\Omega\right) r_{x}\right) d x & \leq \int_{S^{1}} r\left(g_{x x}^{*}+\left(\left(\psi_{x}+\Omega\right) g^{*}\right)_{x}\right) d x .
\end{aligned}
$$

As $g^{*}$ solves (4.4), we obviously have

$$
g_{x x}^{*}+\left(\left(\psi_{x}+\Omega\right) g^{*}\right)_{x}=(\Omega-\omega) g_{x}^{*}
$$

whence

$$
\frac{d}{d t} \Sigma[g] \leq(\Omega-\omega) \int_{S^{1}} r g_{x}^{*} d x=(\Omega-\omega) \int_{S^{1}} g \frac{g_{x}^{*}}{g^{*}} d x
$$

and using the conservation of mass once again, we obtain

$$
\frac{d}{d t} \Sigma[g] \leq M_{1}|\Omega-\omega|
$$

This yields

$$
\Sigma[g(\cdot, \tau)] \leq M_{1} \tau|\Omega-\omega|+\Sigma[g(\cdot, 0)]
$$

whenever $\Sigma[g(\cdot, 0)]$ exists. Using (4.3), we can write the last inequality in the form

$$
\Sigma[g(\cdot, \tau)] \leq M_{1} \omega T+\Sigma[g(\cdot, 0)]
$$


Now for $t \in[\tau, T]$ the function $g$ solves equation (4.19) and $g^{*}$ is the stationary solution of the same equation, so by Corollary 2.9

$$
\Sigma[g] \leq \Sigma[g(\cdot, \tau)] \mathrm{e}^{-\gamma(t-\tau)} .
$$

From this inequality and (4.22) we get

$$
\Sigma[g(\cdot, T)] \leq\left(M_{1} \omega T+\Sigma[g(\cdot, 0)]\right) \mathrm{e}^{-\gamma(T-\tau)} .
$$

Denote by $\mathcal{T}$ the operator taking a probability density $u \in L^{1}\left(S^{1}\right)$ to $g(\cdot, T)$, where $g$ solves (4.18) -(4.19) with the initial condition $\left.g\right|_{t=0}=u$, and put

$$
R=\frac{M_{1} \omega T}{\mathrm{e}^{\gamma(T-\tau)}-1}
$$

It follows from (4.23) that $\mathcal{T}$ maps the set

$$
X=\left\{u \in W_{2}^{1}\left(S^{1}\right) \cap\{\text { probability densities }\} \mid \Sigma[u] \leq R\right\}
$$

into itself. Moreover, $X$ is a convex closed subset of $W_{2}^{1}\left(S^{1}\right)$ and by parabolic regularity $\mathcal{T}: X \rightarrow X$ is continuous and $\mathcal{T}(X)$ is precompact in $W_{2}^{1}\left(S^{1}\right)$. Hence by the Schauder fixed point theorem $\mathcal{T}$ has a fixed point in $X$, which is the initial data for a time-periodic solution of (4.18)-(4.19). However, such periodic solution is unique, so the fixed point found above coincides with $g_{\infty}(\cdot, 0)$. Therefore, $\Sigma\left[g_{\infty}(\cdot, 0)\right] \leq R$ and (4.21) follows from (4.22).

Proof of Theorem 4.4. We have:

$$
\begin{aligned}
& \left|v_{\infty}(\omega, T, \tau)-v_{\infty \infty}(\omega)\right| \\
& =\left|-\frac{1}{T} \int_{0}^{T} \int_{S^{1}} \psi_{x} g_{\infty} d x d t+\int_{S^{1}} \psi_{x} g^{*} d x\right| \\
& \quad \leq \frac{1}{T}\left|\int_{0}^{\tau} \int_{S^{1}} \psi_{x} g_{\infty} d x d t\right| \\
& +\frac{1}{T}\left|\int_{\tau}^{T} \int_{S^{1}} \psi_{x}\left(g_{\infty}-g^{*}\right) d x d t\right|+\frac{\tau}{T}\left|\int_{S^{1}} \psi_{x} g^{*} d x\right| \\
& \leq \frac{\tau}{T}\left(M_{0}+v_{\infty}(\omega)\right)+\frac{M_{0}}{T} \int_{\tau}^{T}\left\|g_{\infty}-g^{*}\right\|_{L^{1}\left(S^{1}\right)} d t
\end{aligned}
$$

(here we have used the conservation of mass for $g_{\infty}$ ). We now estimate the last term. Using the Csiszár-Kullback inequality and the 
attraction (2.31), we have

$$
\begin{aligned}
& \frac{M_{0}}{T} \int_{\tau}^{T}\left\|g_{\infty}-g^{*}\right\|_{L^{1}\left(S^{1}\right)} d t \leq \frac{M_{0}}{T} \int_{\tau}^{T} \sqrt{2 \Sigma\left[g_{\infty}\right]} d t \\
& \quad \leq \frac{M_{0}}{T} \sqrt{2 \Sigma\left[g_{\infty}(\cdot, \tau)\right]} \int_{\tau}^{T} \mathrm{e}^{-\gamma(t-\tau) / 2} d t \leq \frac{1}{T} \frac{2 M_{0}}{\gamma} \sqrt{2 \Sigma\left[g_{\infty}(\cdot, \tau)\right]} .
\end{aligned}
$$

Estimating the entropy $\Sigma\left[g_{\infty}(\cdot, \tau)\right]$ by means of (4.21), we have

$$
\frac{M_{0}}{T} \int_{\tau}^{T}\left\|g_{\infty}-g_{*}\right\|_{L^{1}\left(S^{1}\right)} d t \leq \frac{1}{T} \frac{2^{3 / 2} M_{0}}{\gamma}\left(M_{1} \omega T\left(1+\frac{1}{\mathrm{e}^{\gamma T(1-\tau / T)}-1}\right)\right)^{\frac{1}{2}} .
$$

Combining this inequality with (4.25), we obtain (4.14).

We finish this section by considering the travelling potential

$$
\Psi(x, t)=\psi(x-\omega t),
$$

where $\psi$ is 1 -periodic and $\omega$ is a constant. The corresponding ratchet model [5, 6] coincides with the one for the stochastic Stokes' drift [4, 21. In [5] it was conjectured that the average drift velocity of this ratchet is positive for positive $\omega$ (cf. also [32, Section 4.4.1]). It is straightforward to check (cf. [6]) that the corresponding periodic solution of (2.7) with $F=-\Psi_{x}$ satisfying (2.11) is

$$
g_{\infty}=g^{*}(x-\omega t),
$$

where $g^{*}$ is defined above in this section. Then the corresponding bulk velocity is

$$
\begin{aligned}
v_{\infty}=-\frac{1}{T} \int_{0}^{T} \int_{0}^{1} \psi_{x}(x-\omega t) g^{*}(x-\omega t) d x d t & \\
= & -\int_{0}^{1} \psi_{x}(x) g^{*}(x) d x \equiv \omega-A(\omega) .
\end{aligned}
$$

The conjecture of [5] (for any non-constant $\psi$ ) thus follows from our Proposition 4.3.

\section{Small Diffusion COEFficient}

We are now interested in applying the bulk velocity representation (2.19) for finding sufficient conditions of transport in the case of small $\sigma$.

The result concerns generic piecewise smooth potentials $\Psi(x, t)$ which are $T$-periodic in $t$ and whose $x$-derivatives are 1-periodic in $x$.

Theorem 5.1. Assume that the ODE

$$
y^{\prime}(t)=F(y(t), t)
$$


(where $F=-\Psi_{x}$ is as in Theorem 2.2) has no T-periodic solutions. Then $v_{\infty} \neq 0$ for sufficiently small $\sigma$. Moreover,

$$
\operatorname{sign} v_{\infty}=\operatorname{sign}(y(T)-y(0))
$$

for every solution y to (5.1).

Proof. Given a sequence $\sigma_{n} \rightarrow 0$, consider the corresponding $T$-periodic solutions $g_{\infty n}$ to (2.7) satisfying (2.11), and the bulk velocities $v_{\infty n}$ defined by (2.19). If the theorem is not true, $\left\{\sigma_{n}\right\}$ can be chosen in such a way that every $v_{\infty n}$ violates (5.2). Consider the following auxiliary equation

$$
\zeta_{t}+(F \zeta)_{x}=0, \quad(t, x) \in(0, \infty) \times \mathbb{R} .
$$

It is easy to see that the solution $\zeta$ of (5.3) can be expressed in the form $\zeta(x, t)=[z(s(x, t))]_{x}$, where $z_{x}(x)=\zeta(x, 0)$, and $s$ solves the problem

$$
\left\{\begin{array}{l}
s_{t}+F s_{x}=0, \\
s(x, 0)=x, \quad x \in \mathbb{R} .
\end{array}\right.
$$

The sequence of corresponding positive periodic solutions $\left\{g_{\infty n}\right\}$ to (2.7), (2.11) is bounded in $L^{\infty}\left(0, T ; L^{1}\left(S^{1}\right)\right)$, and $\left\{\frac{\partial g_{\infty n}}{\partial t}\right\}$ is bounded in $L^{\infty}\left(0, T ; W_{1}^{-2}\left(S^{1}\right)\right)$, so, by the Aubin-Lions-Simon lemma, without loss of generality, $g_{\infty n}$ converges strongly in $C\left([0, T] ; H^{-1}\left(S^{1}\right)\right)$ to some $\zeta_{\infty}$. Due to (2.19), for large $n$, the sign of $v_{\infty n}$ coincides with the sign of

$$
v_{\infty 0}=\frac{1}{T} \int_{0}^{T}\left\langle\zeta_{\infty}(\cdot, t), F(\cdot, t)\right\rangle_{H^{-1}\left(S^{1}\right) \times H^{1}\left(S^{1}\right)} d x d t
$$

provided $v_{\infty 0} \neq 0$.

Let $z_{\infty} \in L_{l o c}^{2}(\mathbb{R})$ be such that $\left(z_{\infty}\right)_{x}(x)=\zeta_{\infty}(x, 0)$ (obviously, $\zeta_{\infty}(\cdot, 0)$ can be considered as a distribution on $\left.\mathbb{R}\right)$. Since $\left\langle g_{\infty n}(\cdot, 0), 1\right\rangle=$ 1 , in the limit we have $\left\langle\zeta_{\infty}(\cdot, 0), 1\right\rangle=1$, so $\left.z_{\infty}\right|_{[0,1]}$ is a non-constant function. The functions $g_{\infty n}$ are positive by Theorem 2.2 , so $\zeta_{\infty}(\cdot, 0) \geq$ 0 in the sense of distributions on $S^{1}$, whence $z_{\infty}$ is essentially a nondecreasing function.

Passing to the limit, we see that $\zeta_{\infty}$ solves (5.3) in the sense of distributions. Hence,

$$
\zeta_{\infty}(x, t)=\left[z_{\infty}(s(x, t))\right]_{x}
$$

and

$$
\begin{aligned}
& {\left[z_{\infty}(s(x, t))\right]_{t}+F(x, t) \zeta_{\infty}(x, t)} \\
& \quad=\left[z_{\infty}(s(x, t))\right]_{t}+F(x, t)\left[z_{\infty}(s(x, t))\right]_{x}=0
\end{aligned}
$$


in the sense of distributions. The second equality is obvious if $z_{\infty}$ is smooth, and the general case follows from the dominated convergence theorem.

But (5.5) and (5.7) yield

$$
\begin{array}{rl}
v_{\infty 0}=-\frac{1}{T} \int_{0}^{T} \int_{0}^{1}\left[z_{\infty}(s(x, t))\right]_{t} & d x d t \\
& =\frac{\int_{0}^{1}\left(z_{\infty}(x)-z_{\infty}(s(x, T)) d x\right.}{T} .
\end{array}
$$

Solving (5.4) by the method of characteristics we infer that $x=y(T)$, where $y(t)$ is the solution of (5.1) with the initial condition $y(0)=$ $s(x, T)$. Since $S^{1}$ is compact and (5.1) has no $T$-periodic solutions, the difference

$$
d(y)=y(T)-y(0)
$$

is separated from zero, and its sign does not change for all solutions of (5.11). Consequently, $v_{\infty 0} \neq 0$ (otherwise $\left.z_{\infty}\right|_{[0,1]}$ would be a constant). We conclude that $v_{\infty 0}$ and $v_{\infty n}$ (for large $n$ ) have the same sign as $d(y)$, reaching a contradiction.

Remark 5.2. Observe that (5.1) has no $T$-periodic solutions if and only if there exists an unbounded trajectory or, in other words, if and only if the corresponding Poincaré's rotation number [10] is nonzero. Hence, the sign of the rotation number coincides with the sign of $v_{\infty 0}$. A related observation was made in [34] for diffusion-free tilting ratchets. Our conjecture is that $\lim _{\sigma \rightarrow 0} v_{\infty}=r / T$, where $r$ is the rotation number (cf. [34]).

Remark 5.3. Existence of periodic orbits is unstable with respect to perturbations of $F$, thus, in a general position, $v_{\infty}$ is non-zero for small $\sigma$. Unfortunately, as R. Ortega and F. Zanolin pointed out in a personal communication, there are no criteria of the form that 'some set of assumptions on $F$ implies non-existence of $T$-periodic solutions to (5.1)'. In [25], the non-existence of periodic solutions to (5.1) with a tilting potential of the form $F_{\sin }(y, t)=H(t)-\sin y$, where $H$ is $T$ periodic and satisfies (2.22), was discovered. There, it was observed that this phenomenon contrasts with the behaviour of the equation

$$
y^{\prime \prime}(t)=F_{\sin }(y(t), t),
$$

which always admits $T$-periodic solutions. However, for this particular class of tilting potentials there is some hope to get a criterion using a trick from [26], transforming (5.1) into a Ricatti equation. The discrete analogue of the problem produces Arnold tongues. 


\section{Multi-State MODELS}

We now consider another class of ratchet models, where the particles can be in several states, and the total amount of particles is fixed. Particles in different states are sensitive to different time-independent potentials. The particles can randomly change their states with rates $\nu_{i j}(x) \geq 0$ (from $j$-th state to $i$-th state; $i, j=1, \ldots, N ; i \neq j$ ), calling forth the bulk transport. This leads to the following general Cauchy problem for a system of Fokker-Planck-type equations:

$$
\left\{\begin{array}{l}
\left(\rho_{i}\right)_{t}-\sigma\left(\rho_{i}\right)_{x x}-\left(\left(\Psi_{i}\right)_{x} \rho_{i}\right)_{x}+\sum_{j, j \neq i} \nu_{j i} \rho_{i}=\sum_{j, j \neq i} \nu_{i j} \rho_{j}, \\
\rho_{i}(x, 0)=\rho_{i 0}(x),
\end{array}\right.
$$

which we again consider for all $x \in \mathbb{R}$ and $t>0$.

Here $\Psi_{i}(x)$ are given $C^{4}$-regular potentials, $\rho_{i_{0}}$ are given initial mass distributions, and $\sigma$ is the diffusion coefficient (for definiteness, we set it to be the same for all states). We assume that $\left(\Psi_{i}\right)_{x}(x)$ and $\nu_{i j}(x)$ are 1-periodic and that $\rho_{i 0}(x)$ satisfy the requirements

$$
\rho_{i 0}(x) \geq 0, \sum_{i=1}^{N} \int_{-\infty}^{\infty} \rho_{i 0}(x) d x=1, \int_{-\infty}^{\infty}|x| \rho_{i 0}(x) d x<\infty .
$$

Standard arguments show that (6.1) has a unique solution for any continuous vector function $\rho_{0}(x)=\left(\rho_{i 0}\right)$ satisfying (6.2); moreover, for any $t>0$, the components $\rho_{i}(x, t)$ of the solution vector $\rho(x, t)$ are positive. Moreover, the classical estimates [14] for the fundamental matrix of solutions to (6.1) imply

$$
\lim _{x \rightarrow \pm \infty}|x|\left(|\rho(x, t)|+\left|\rho_{x}(x, t)\right|\right)=0 .
$$

Let

$$
\tilde{\rho}(x, t)=\sum_{i=1}^{N} \rho_{i}(x, t)
$$

be the total density function. Adding the equations of (6.1) gives

$$
\tilde{\rho}_{t}-\sigma \tilde{\rho}_{x x}-\sum_{i=1}^{N}\left(\left(\Psi_{i}\right)_{x} \rho_{i}\right)_{x}=0
$$

As in Section 2, (6.3) and (6.5) yield conservation of the total mass

$$
\int_{-\infty}^{\infty} \tilde{\rho}(x, t) d x=1
$$


and finiteness of the centre of mass

$$
\bar{x}(t)=\int_{-\infty}^{\infty} x \tilde{\rho}(x, t) d x
$$

for any $t$. Similarly to Section 2, we are interested in the properties of the velocity of the centre of mass $\bar{x}(t)$.

We consider the following auxiliary problem on $S^{1} \times(0, \infty)$ :

$$
\left\{\begin{array}{l}
\left(g_{i}\right)_{t}-\sigma\left(g_{i}\right)_{x x}-\left(\left(\Psi_{i}\right)_{x} g_{i}\right)_{x}+\sum_{j, j \neq i} \nu_{j i} g_{i}=\sum_{j, j \neq i} \nu_{i j} g_{j}, \\
g_{i}(x)>0, \sum_{i=1}^{N} \int_{S^{1}} g_{i}(x) d x=1
\end{array}\right.
$$

Observe that if a vector $\rho$ with positive components solves (6.1), then

$$
g(x, t)=\sum_{k=-\infty}^{\infty} \rho(x+k, t)
$$

solves (6.8). The convergence of this series follows from the properties of the fundamental matrix of solutions to (6.1).

Then the velocity of the centre of mass is

$$
\begin{aligned}
\tilde{v}(t):=\frac{\partial \bar{x}}{\partial t}(t)=\int_{-\infty}^{\infty} x \tilde{\rho}_{t}(x, t) d x & \\
= & \int_{-\infty}^{\infty} x\left(\sigma \tilde{\rho}_{x x}+\sum_{i=1}^{N}\left(\left(\Psi_{i}\right)_{x} \rho_{i}\right)_{x}\right) d x \\
& =-\int_{-\infty}^{\infty}\left(\sigma \tilde{\rho}_{x}+\sum_{i=1}^{N}\left(\Psi_{i}\right)_{x} \rho_{i}\right) d x \\
& =-\sum_{i=1}^{N} \int_{0}^{1}\left(\Psi_{i}\right)_{x} g_{i} d x
\end{aligned}
$$

We need the following result on existence of a unique attractor for (6.8).

Proposition 6.1. There exists a unique regular stationary solution vector $g_{\infty}(x)=\left(g_{i \infty}\right)$ to (6.8). Moreover, there exists $\gamma>0$ such that, for any smooth solution $g$ to (6.8), there exists a positive constant $C=C(g)$ such that

$$
\left|g(x, t)-g_{\infty}(x)\right| \leq C \mathrm{e}^{-\gamma t}, \quad(x, t) \in S^{1} \times(0, \infty) .
$$

Proof. The existence and uniqueness can be proved similarly to [9, Theorem 4.1], and the stability follows in the same way as in [20, Theorem 4.1]. 
We can now put

$$
\tilde{v}_{\infty}:=-\sum_{i=1}^{N} \int_{0}^{1}\left(\Psi_{i}\right)_{x}(x) g_{i \infty}(x) d x,
$$

arriving at the following bound.

Corollary 6.2. For any solution of (6.1), we have

$$
\left|\tilde{v}(t)-\tilde{v}_{\infty}\right| \leq C e^{-\gamma t}
$$

where $C$ depends only on $\rho_{0}$.

Thus, the sign of the asymptotic velocity $\tilde{v}_{\infty}$ determines the direction of transport. We now examine several situations when $\tilde{v}_{\infty}$ can be proved to be non-zero. For simplicity, we restrict ourselves to the two-state models. We begin with the small diffusion case.

Theorem 6.3. Let $N=2$. Suppose that the functions $F_{1}=-\left(\Psi_{1}\right)_{x}$ and $F_{2}=-\left(\Psi_{2}\right)_{x}$ merely have a finite number of zeros on $S^{1}$, and do not admit common zeros. Let $\sigma$ be sufficiently small, $\nu_{12}(x)>0$, $\nu_{21}(x)>0$.

i) Assume that both $F_{1}$ and $F_{2}$ have zeros, and for every $x_{*}$ such that $F_{1}\left(x_{*}\right) F_{2}\left(x_{*}\right)=0$, let $i_{*}=1$ if $F_{2}\left(x_{*}\right)=0$, and $i_{*}=2$ if $F_{1}\left(x_{*}\right)=0$. Then

$$
\operatorname{sign} \tilde{v}_{\infty}=\operatorname{sign} F_{i_{*}}\left(x_{*}\right),
$$

provided the sign in the right-hand side of (6.14) is independent of $x_{*}$.

ii) Assume that only one of the functions $F_{1}$ and $F_{2}$ possesses zeros. Let $F_{i_{*}}$ be the potential gradient which does not have zeros. Then

$$
\operatorname{sign} \tilde{v}_{\infty}=\operatorname{sign} F_{i_{*}} .
$$

iii) Assume that none of the functions $F_{1}$ and $F_{2}$ vanishes. Then

$$
\operatorname{sign} \tilde{v}_{\infty}=\operatorname{sign}\left(F_{1} F_{2}\right) \operatorname{sign} \int_{0}^{1}\left(\frac{\nu_{12}(x)}{F_{2}(x)}+\frac{\nu_{21}(x)}{F_{1}(x)}\right) d x,
$$

provided the integral in the right-hand side is non-zero.

Remark 6.4. In the case when $F_{1}$ and $F_{2}$ have common zeros or the sign in the right-hand side of (6.14) varies, the transport still can be present, but more subtle methods are required to establish that $\tilde{v}_{\infty} \neq 0$.

Remark 6.5. In case i), if the sign in (6.14) is positive, it is simple to see that $\max \left(F_{1}, F_{2}\right)>0$, and there is an interval where $\min \left(F_{1}, F_{2}\right)>$ 0 . Hence, the occurrence of unidirectional transport in the Neumann problem setting on a bounded segment follows from [29, Theorem 1] or 
[20, Theorem 3.1]. The cases ii) and iii) are not covered by the results of [8, 20, 29].

Proof of Theorem 6.3. Assume there is a sequence $\sigma_{n} \rightarrow 0$ such that the corresponding velocities $\tilde{v}_{\infty n}$ violate (6.14), or (6.15), or (6.16), respectively.

The sequence of stationary solutions $\left\{g_{\infty n}\right\}$ is bounded in $\left[L^{1}\left(S^{1}\right)\right]^{2}$, so, without loss of generality, $g_{\infty n}$ converges weakly-* in $\left[C^{*}\left(S^{1}\right)\right]^{2}$ to some $\zeta$. For large $n$, the sign of $\tilde{v}_{\infty n}$ coincides with the sign of

$$
\tilde{v}_{\infty 0}=\left\langle\zeta_{1}, F_{1}\right\rangle_{C^{*} \times C}+\left\langle\zeta_{2}, F_{2}\right\rangle_{C^{*} \times C},
$$

provided $\tilde{v}_{\infty 0} \neq 0$. Observe that $\zeta$ solves the system

$$
\left\{\begin{array}{l}
\left(F_{1} \zeta_{1}\right)_{x}=\nu_{12} \zeta_{2}-\nu_{21} \zeta_{1} \\
\left(F_{2} \zeta_{2}\right)_{x}=\nu_{21} \zeta_{1}-\nu_{12} \zeta_{2} \\
\zeta_{i} \geq 0,\left\langle\zeta_{1}+\zeta_{2}, 1\right\rangle=1
\end{array}\right.
$$

in the sense of distributions on $S^{1}$. Adding these two equations, we see that $F_{1} \zeta_{1}+F_{2} \zeta_{2}$ is essentially a constant, and, due to (6.17),

$$
F_{1} \zeta_{1}+F_{2} \zeta_{2}=\tilde{v}_{\infty 0}
$$

in the sense of distributions.

Consider first cases i) and ii). The measures $\zeta_{1}$ and $\zeta_{2}$ cannot be concentrated only at the zeros of $F_{1} F_{2}$. Indeed, if that is so, then (6.19) implies

$$
F_{1} \zeta_{1}=F_{2} \zeta_{2}=0
$$

in the sense of distributions, so the supports of $\zeta_{1}$ and $\zeta_{2}$ are disjoint. But (6.18) gives $\nu_{12} \zeta_{2}=\nu_{21} \zeta_{1}$, so the supports should coincide, and we get a contradiction.

Hence, there are two adjacent zeros $x_{1}$ and $x_{2}$ of $F_{1} F_{2}$ such that the support of $\zeta_{1}$ or $\zeta_{2}$ intersects with the interval $\left(x_{1}, x_{2}\right)$ (in the case when $F_{1} F_{2}$ has only one zero, we can change the variable $x$ for $\tilde{x}=x / 2$ in (6.1), which doubles the number of zeros on $S^{1}$, but does not affect the transport direction).

Suppose that $\tilde{v}_{\infty 0}=0$. Then the solution of the system (6.18), (6.19) on $\left(x_{1}, x_{2}\right)$ may be written explicitly, with an unknown multiplicative constant $M \neq 0$,

$$
\left\{\begin{array}{l}
\zeta_{1}(x)=M \exp \left(\int_{x}^{\bar{x}} \frac{\nu_{12}(y)}{F_{2}(y)}+\frac{\nu_{21}(y)}{F_{1}(y)} d y\right) / F_{1}(x), \\
\zeta_{2}(x)=-M \exp \left(\int_{x}^{\bar{x}} \frac{\nu_{12}(y)}{F_{2}(y)}+\frac{\nu_{21}(y)}{F_{1}(y)} d y\right) / F_{2}(x),
\end{array}\right.
$$

where $\bar{x}=\frac{x_{1}+x_{2}}{2}$. This gives that $F_{1} F_{2}$ is negative on $\left(x_{1}, x_{2}\right)$. Consequently, $x_{1}$ and $x_{2}$ are zeros of the same potential - in case ii) this is 
trivial - and, for definiteness, let it be $F_{1}$. Observe that one of the integrals $\int_{x_{1}}^{\bar{x}} \frac{\nu_{12}(y)}{F_{2}(y)}+\frac{\nu_{21}(y)}{F_{1}(y)} d y$ and $\int_{x_{2}}^{\bar{x}} \frac{\nu_{12}(y)}{F_{2}(y)}+\frac{\nu_{21}(y)}{F_{1}(y)} d y$ is positive. Since $F_{1}$ is $C^{1}$-smooth, $\int_{x_{1}}^{x_{2}} \frac{1}{F_{1}(y)} d y= \pm \infty$. Hence, $\left.\int_{x_{1}}^{x_{2}} \zeta_{1}\right|_{\left(x_{1}, x_{2}\right)}(y) d y=\infty$. But (6.18) implies $\left.\int_{x_{1}}^{x_{2}} \zeta_{1}\right|_{\left(x_{1}, x_{2}\right)}(y) d y \leq 1$, and we arrive at a contradiction.

Assume now that $F_{1}>0$ on $\left(x_{1}, x_{2}\right)$. We then have to show that $\tilde{v}_{\infty 0}<0$. One can check that, under the assumptions that we have made, the only solution of (6.18), (6.19) with finite integral on $\left(x_{1}, x_{2}\right)$ is

$$
\left\{\begin{array}{l}
\zeta_{1}(x)=\frac{\tilde{v}_{\infty 0}}{F_{1}(x)} \int_{x_{1}}^{x} \exp \left(\int_{x}^{s} \frac{\nu_{12}(y)}{F_{2}(y)}+\frac{\nu_{21}(y)}{F_{1}(y)} d y\right) \frac{\nu_{12}(s)}{F_{2}(s)} d s \\
\zeta_{2}(x)=\frac{\tilde{v}_{\infty 0}}{F_{2}(x)}\left[1-\int_{x_{1}}^{x} \exp \left(\int_{x}^{s} \frac{\nu_{12}(y)}{F_{2}(y)}+\frac{\nu_{21}(y)}{F_{1}(y)} d y\right) \frac{\nu_{12}(s)}{F_{2}(s)} d s\right]
\end{array}\right.
$$

So $\zeta_{1} \geq 0$ and $\left.F_{2}\right|_{\left(x_{1}, x_{2}\right)}<0$ imply $\tilde{v}_{\infty 0}<0$. The case $F_{1}<0$ is treated similarly.

In case iii), the solution $\zeta$ is smooth on the whole circle $S^{1}$, so it may be identified with a 1 -periodic function on $\mathbb{R}$. Moreover, it has the form

$$
\begin{aligned}
\zeta_{1}(x)= & \frac{M}{F_{1}(x)} \exp \left(\int_{x}^{0} \frac{\nu_{12}(y)}{F_{2}(y)}+\frac{\nu_{21}(y)}{F_{1}(y)} d y\right) \\
& +\frac{\tilde{v}_{\infty 0}}{F_{1}(x)} \int_{0}^{x} \exp \left(\int_{x}^{s} \frac{\nu_{12}(y)}{F_{2}(y)}+\frac{\nu_{21}(y)}{F_{1}(y)} d y\right) \frac{\nu_{12}(s)}{F_{2}(s)} d s \\
\zeta_{2}(x)= & -\frac{M}{F_{2}(x)} \exp \left(\int_{x}^{0} \frac{\nu_{12}(y)}{F_{2}(y)}+\frac{\nu_{21}(y)}{F_{1}(y)} d y\right) \\
+ & \frac{\tilde{v}_{\infty 0}}{F_{2}(x)}\left[1-\int_{0}^{x} \exp \left(\int_{x}^{s} \frac{\nu_{12}(y)}{F_{2}(y)}+\frac{\nu_{21}(y)}{F_{1}(y)} d y\right) \frac{\nu_{12}(s)}{F_{2}(s)} d s\right]
\end{aligned}
$$

Due to periodicity,

$$
\begin{aligned}
& M\left[\exp \left(\int_{1}^{0} \frac{\nu_{12}(y)}{F_{2}(y)}+\frac{\nu_{21}(y)}{F_{1}(y)} d y\right)-1\right] \\
& \quad+\tilde{v}_{\infty 0} \int_{0}^{1} \exp \left(\int_{x}^{s} \frac{\nu_{12}(y)}{F_{2}(y)}+\frac{\nu_{21}(y)}{F_{1}(y)} d y\right) \frac{\nu_{12}(s)}{F_{2}(s)} d s=0 .
\end{aligned}
$$

Hence, $\tilde{v}_{\infty 0} \neq 0$, for the contrary would imply $M=0$, and $\zeta \equiv 0$, which contradicts (6.18).

Assume that the right-hand side of (6.16) is positive, and $\tilde{v}_{\infty 0}$ is negative (the opposite situation is completely analogous). Then at least one of the potentials, say $F_{1}$, is positive. Due to the positivity of 
the right-hand side of (6.16),

$$
\operatorname{sign}\left(\exp \left(\int_{1}^{0} \frac{\nu_{12}(y)}{F_{2}(y)}+\frac{\nu_{21}(y)}{F_{1}(y)} d y\right)-1\right)=-\operatorname{sign} F_{2},
$$

and (6.25) yields

$$
\operatorname{sign} M=\operatorname{sign} \tilde{v}_{\infty 0}
$$

It follows from (6.23) and (6.27) that $\zeta_{1}(0)<0$, which contradicts the positivity of the solution.

We now address the transport properties of the randomly tilting ratchet, i.e., the model of the form

$$
\left\{\begin{array}{l}
\left(\rho_{1}\right)_{t}-\left(\rho_{1}\right)_{x x}-\left(\left[\psi_{x}+\omega\right] \rho_{1}\right)_{x}+\nu_{21} \rho_{1}=\nu_{12} \rho_{2}, \\
\left(\rho_{2}\right)_{t}-\left(\rho_{2}\right)_{x x}-\left(\left[\psi_{x}+\Omega\right] \rho_{2}\right)_{x}+\nu_{12} \rho_{2}=\nu_{21} \rho_{1}, \\
\rho(x, 0)=\rho_{0}(x),
\end{array}\right.
$$

where $\psi(x)$ is a $C^{4}$-smooth 1 -periodic potential, the diffusion coefficient $\sigma$ is taken to be $1 ; \omega, \Omega, \nu_{12}>0, \nu_{21}>0$ are scalars (independent of $x$, for simplicity), and $\rho_{0}$ satisfies (6.2). We assume the following non-bias condition (cf. (4.3)) :

$$
\Omega \nu_{21}+\omega \nu_{12}=0
$$

Denote by $\tilde{v}_{\infty}(\omega, \nu)$ the corresponding bulk velocity defined by (6.12).

The following theorem shows that the adiabatic and semi-adiabatic bulk velocities of the randomly tilting ratchet are the same as for the tilting ratchet. Thus, the results of the previous sections may be applied to determine the transport direction. In particular, the direction of the semiadiabatic transport is determined by the sign of $\omega$ for every non-constant $\psi$.

Theorem 6.6. Let $\omega$ be fixed. Then

$$
\begin{gathered}
\lim _{\nu_{12}=\nu_{21} \rightarrow 0} \tilde{v}_{\infty}(\omega, \nu)=-\frac{A(\omega)+A(-\omega)}{2} ; \\
\lim _{\substack{\nu_{21} \rightarrow 0 \\
\nu_{21} / \nu_{12} \rightarrow 0}} \tilde{v}_{\infty}(\omega, \nu)=\omega-A(\omega) .
\end{gathered}
$$

The proof is left as an exercise for the reader.

\section{REFERENCES}

[1] R. Ait-Haddou and W. Herzog. Brownian ratchet models of molecular motors. Cell biochemistry and biophysics, 38(2):191-213, 2003.

[2] R. D. Astumian. Thermodynamics and kinetics of a brownian motor. Science, 276(5314):917-922, 1997. 
[3] J.-P. Bartier, J. Dolbeault, R. Illner, and M. Kowalczyk. A qualitative study of linear drift-diffusion equations with time-dependent or degenerate coefficients. Mathematical Models and Methods in Applied Sciences, 17(03):327-362, 2007.

[4] I. Bena, M. Copelli, and C. Van den Broeck. Stokes' drift: A rocking ratchet. Journal of Statistical Physics, 101(1-2):415-424, 2000.

[5] A. Blanchet, J. Dolbeault, and M. Kowalczyk. Travelling fronts in stochastic Stokes' drifts. Physica A: Statistical Mechanics and its Applications, 387(23):5741-5751, 2008.

[6] A. Blanchet, J. Dolbeault, and M. Kowalczyk. Stochastic Stokes' drift, homogenized functional inequalities, and large time behavior of Brownian ratchets. SIAM J. Math. Anal., 41(1):46-76, 2009.

[7] W. R. Browne and B. L. Feringa. Making molecular machines work. Nature nanotechnology, 1(1):25-35, 2006.

[8] M. Chipot, S. Hastings, and D. Kinderlehrer. Transport in a molecular motor system. ESAIM: Mathematical Modelling and Numerical Analysis, 38(06):1011-1034, 2004.

[9] M. Chipot, D. Hilhorst, D. Kinderlehrer, and M. Olech. Contraction in $L^{1}$ for a system arising in chemical reactions and molecular motors. Differ. Equ. Appl., 1(1):139-151, 2009.

[10] E. A. Coddington and N. Levinson. Theory of ordinary differential equations. McGraw-Hill Book Company, Inc., New York-Toronto-London, 1955.

[11] I. Csiszár. $I$-divergence geometry of probability distributions and minimization problems. Ann. Probability, 3:146-158, 1975.

[12] A.-L. Dalibard. Stability of periodic stationary solutions of scalar conservation laws with space-periodic flux. Journal of the European Mathematical Society, 13(5):1245-1288, 2011.

[13] J. Dolbeault, D. Kinderlehrer, and M. Kowalczyk. Remarks about the flashing ratchet. In Partial differential equations and inverse problems, volume 362 of Contemp. Math., pages 167-175. Amer. Math. Soc., Providence, RI, 2004.

[14] S. D. Eidelman. Parabolic systems. North Holland Pub. Co., 1969.

[15] M. Fistul. Symmetry broken motion of a periodically driven brownian particle: Nonadiabatic regime. Physical Review E, 65(4):046621, 2002.

[16] M. Gori. Lower semicontinuity and relaxation for integral and supremal functionals. PhD thesis, University of Pisa, Apr. 2004.

[17] N. Grunewald, F. Otto, C. Villani, and M. G. Westdickenberg. A two-scale approach to logarithmic Sobolev inequalities and the hydrodynamic limit. Ann. Inst. Henri Poincaré Probab. Stat., 45(2):302-351, 2009.

[18] P. Hänggi and F. Marchesoni. Artificial brownian motors: Controlling transport on the nanoscale. Reviews of Modern Physics, 81(1):387, 2009.

[19] P. Hänggi, F. Marchesoni, and F. Nori. Brownian motors. Annalen der Physik, 14(1-3):51-70, 2005.

[20] S. Hastings, D. Kinderlehrer, and J. B. McLeod. Diffusion mediated transport in multiple state systems. SIAM J. Math. Anal., 39(4):1208-1230, 2007.

[21] K. M. Jansons and G. Lythe. Stochastic Stokes drift. Physical Review Letters, 81(15):3136, 1998.

[22] E. Kay, D. Leigh, and F. Zerbetto. Synthetic molecular motors and mechanical machines. Angewandte Chemie International Edition, 46(1-2):72-191, 2007. 
[23] D. Kinderlehrer and M. Kowalczyk. Diffusion-mediated transport and the flashing ratchet. Arch. Ration. Mech. Anal., 161(2):149-179, 2002.

[24] S. Mirrahimi and P. E. Souganidis. A homogenization approach for the motion of motor proteins. Nonlinear Differential Equations and Applications NoDEA, 20(1):129-147, 2013.

[25] R. Ortega. A counterexample for the damped pendulum equation. Acad. Roy. Belg. Bull. Cl. Sci. (5), 73(10):405-409, 1987.

[26] R. Ortega and M. Tarallo. Degenerate equations of pendulum-type. Commun. Contemp. Math., 2(2):127-149, 2000.

[27] J. Parrondo and B. J. de Cisneros. Energetics of brownian motors: a review. Applied Physics A, 75(2):179-191, 2002.

[28] B. Perthame and P. E. Souganidis. Asymmetric potentials and motor effect: a homogenization approach. Ann. Inst. H. Poincaré Anal. Non Linéaire, 26(6):2055-2071, 2009.

[29] B. Perthame and P. E. Souganidis. Asymmetric potentials and motor effect: a large deviation approach. Arch. Ration. Mech. Anal., 193(1):153-169, 2009.

[30] B. Perthame and P. E. Souganidis. A homogenization approach to flashing ratchets. Nonlinear Differential Equations and Applications NoDEA, 18(1):45$58,2011$.

[31] F. O. Porper and S. D. Ėŭdel'man. Two-sided estimates of the fundamental solutions of second-order parabolic equations and some applications of them. Uspekhi Mat. Nauk, 39(3(237)):107-156, 1984.

[32] P. Reimann. Brownian motors: noisy transport far from equilibrium. Physics Reports, 361(2):57-265, 2002.

[33] P. Reimann and P. Hänggi. Introduction to the physics of brownian motors. Applied Physics A, 75(2):169-178, 2002.

[34] R. Salgado-García, M. Aldana, and G. Martínez-Mekler. Deterministic ratchets, circle maps, and current reversals. Physical review letters, 96(13):134101, 2006 .

[35] J. Serrin. On the definition and properties of certain variational integrals. Trans. Amer. Math. Soc., 101:139-167, 1961.

[36] D. Vorotnikov. The flashing ratchet and unidirectional transport of matter. Discrete Contin. Dyn. Syst. Ser. B, 16(3):963-971, 2011.

[37] D. Vorotnikov. Analytical aspects of the Brownian motor effect in randomly flashing ratchets. J. Math. Biol., 68(7):1677-1705, 2014.

(S. Kondratyev) CMUC, Department of Mathematics, University of Coimbra, 3001-501 Coimbra, Portugal

E-mail address: kondratyev@mat.uc.pt

(J.M. Urbano) CMUC, Department of Mathematics, University of Coimbra, 3001-501 Coimbra, Portugal

E-mail address: jmurb@mat.uc.pt

(D. Vorotnikov) CMUC, Department of Mathematics, University of Coimbra, 3001-501 Coimbra, Portugal

E-mail address: mitvorot@mat.uc.pt 